\title{
A check-list of longicorn beetles (Coleoptera: Cerambycidae) of Tyumenskaya Oblast of Russia
}

\section{Аннотированный список жкуков-усачей (Coleoptera: Cerambycidae) Тюменской области}

\author{
V.A. Stolbov*, E.V. Sergeeva**, D.E. Lomakin*, S.D. Sheykin* \\ В.А. Столбов*, Е.В. Сергеева**, А.Е. Аомакин*, С.А. Шейкин* \\ * Tyumen state university, Volodarskogo Str. 6, Tyumen 625003 Russia. E-mail: vitusstgu@mail.ru. \\ * Тюменский государственный университет, ул. Володарского 6, Тюмень 625003 Россия. \\ ** Tobolsk complex scientific station of the UB of the RAS, Acad. Yu. Osipova Str. 15, Tobolsk 626152 Russia. E-mail: \\ elenatbs@rambler.ru. \\ ** Тобольская комплексная научная станция УрО РАН, ул. акад. Ю. Осипова 15, Тобольск 626152 Россия.
}

Key words: Coleoptera, Cerambycidae, Tyumenskaya Oblast, fauna, West Siberia.

Ключевые слова: жесткокрылые, усачи, Тюменская область, фауна, Западная Сибирь.

\begin{abstract}
A checklist of 99 Longhorn beetle species (Cerambycidae) from 59 genera occurring in Tyumenskaya Oblast of Russia, compiled on the basis of author's material, museum collections and literature sources, is presented. Eleven species, Dinoptera collaris (Linnaeus, 1758), Pachytodes erraticus (Dalman, 1817), Stenurella bifasciata (Müller, 1776), Tetropium gracilicorne Reitter, 1889, Spondylis buprestoides (Linnaeus, 1758), Pronocera sibirica (Gebler, 1848), Semanotus undatus (Linnaeus, 1758), Monochamus impluviatus (Motschulsky, 1859), Lamia textor (Linnaeus, 1758), Exocentrus lusitanus (Linnaeus, 1767) and Phytoecia coerulescens (Scopoli, 1763), are recorded for the region for the first time.
\end{abstract}

Резюме. В работе представлен аннотированный список жуков-усачей (Cerambycidae) Тюменской области, основанный на материалах авторов, музейных коллекций и литературных данных. Выявлено 99 видов из 59 родов. Одиннаядцатьвидов: Dinoptera collaris (Linnaeus, 1758), Pachytodes erraticus (Dalman, 1817), Stenurella bifasciata (Müller, 1776), Tetropium gracilicorne Reitter, 1889 , Spondylis buprestoides (Linnaeus, 1758), Pronocera sibirica (Gebler, 1848), Semanotus undatus (Linnaeus, 1758), Monochamus impluviatus (Motschulsky, 1859), Lamia textor (Linnaeus, 1758), Exocentrus lusitanus (Linnaeus, 1767) и Phytoecia coerulescens (Scopoli, 1763)для региона приводятся впервые.

\section{Introduction}

The fauna of Longicorn beetles (Cerambycidae) of Russia is well studied and, at present, includes 586 species [Danilevsky, 2019]. However, not all regions are well investigated, among them the vast territory of Western Siberia. The most extensive information on the species diversity of this family in Western Siberia is given in a series of keys to species of Longicorns of North Asia [Cherepanov, 1979, 1981, 1982, 1983, 1984, 1985], based mainly on collections from Tomskaya oblast, Novosibirskaya oblast and Altai. Research that is more contemporary includes only a list of 96 species of Ce- rambycidae of Tomskaya oblast [Kuleshov, Romanenko, 2009].

The data on the fauna of longicorn beetles of the Tyumenskaya oblast are fragmentary. Ernest Chiki gave the first references of the Cerambycidae of Tyumen oblast at the beginning of the XX century. He indicated 11 species and noted in general the northern character of the enthomofauna of the region [Csíki, 1901]. Later, several species were mentioned in the articles of Yu.M. Kolosov [1914, 1928, 1933] and K.P. Samko [1928a, b], as well as in the later articles of N.N. Plavilshchikov [1936, 1940] and A.I. Cherepanov [1970, 1979, 1981]. In 80-ies and 90-ies XX century the beetles were studied, including Cerambycidae, of Polar, Nether-Polar Ural, lower reaches of the $\mathrm{Ob}$ and Yamal peninsula [Olshvang, 1977, 1980, 1992; Ryabitsev, 1997; Zinovyev, Olshvang, 2003]. Since 1998, rare and interesting species of Longicorns have been investigated [Sitnikov, 1998, 2000, 2002, 2010, 2013; Sitnikov et al., 2004, 2013; Tolstikov et al., 2013; Stolbov et al., 2017], some of them were included in the 1-st edition of the Red Data Book of the Tyumen oblast [2004]. Also, Longicorn beetles were studied in some protected areas [Zinovyev, Ryabitsev, 2000; Zinovyev, Malozemov, 2002; Zinovyev, Nesterkov, 2003; Zinovyev, 2005, 2008; Koltunov et al., 2009; Ukhova, 2010]. In 2011 as a result of many years' research a checklist of the beetles has been published of southern taiga zone, including 60 species of Cerambycidae [Bukhkalo et al., 2011].

Tyumenskaya oblast located on the border with the Urals, is interesting from the faunistic point of view, as the region along which are located the borders of the ranges of European and Siberian species, including twin species, the boundaries of which are still not completely understood [Lazarev, 2009; Danilevsky, 2014]. Thus, investigating of these species in the studied territory is very important for zoogeography and to understand their speciation processes. 


\section{Material and methods}

The basis of this research is formed by the many years' collections of authors in the territory of the Tyumen oblast. Beetles were collected using traditional methods of entomological research. The authors studied the collections of the Tobolsk Complex Scientific Station of the Russian Academy of Sciences, Tobolsk Historical and Architectural Museum-Reserve, Museum Complex of Slovtsov (Tyumen), Zoological Museum of Tyumen State University, Museum of Institute of Plant and Animal Ecology of the Russian Academy of Sciences (Yekaterinburg) and private collections. The identification of most species was carried out by the authors of the article, also by M.L. Danilevsky (A.N. Severtsov Institute of Ecology and Evolution, Moscow) for some doubtful species. All literature, including rare regional editions, critically examined and analyzed.

The list contains literary references for each species, as well as data on general information and regional distribution. Detailed label data is given only for rare species, known from single finds. For other species the nearest inhabited localities are indicated (listed from north to south).

Nomenclature and general information on the distribution of species is adopted according to the catalog of M.L. Danilevsky [2019].

The following abbreviations are used in the text: $\boldsymbol{A S}$ - Administrative South of Tyumenskaya oblast, $\boldsymbol{I P A E}$ - Institute of Plant and Animal Ecology of Russian Academy of Sciences (Yekaterinburg), $\boldsymbol{K} \boldsymbol{H} \boldsymbol{M A O}$ Khanty-Mansi Autonomous Okrug, MK - Museum Complex of Slovtsov (Tyumen), $\mathbf{N M}$ - Natural monument, $\boldsymbol{N P}$ - Nature Park, $\boldsymbol{N} \boldsymbol{R}$ - Nature Reserve, $\boldsymbol{Y A N A O}$ - Yamalo-Nenets Autonomous Okrug, $\boldsymbol{Z I N}$ Zoological Institute of Russian Academy of Sciences (St. Petersburg). New species for the Tyumenskaya oblast are marked with $(*)$, species known only from literary data are marked with $(* *)$.

\section{List of species}

Cerambycidae Latreille, 1802

Prioninae Latreille, 1802

Meroscelisini

Tragosoma depsarium (Linnaeus, 1767)

Tragosoma depsarium: Tobolsk [Kolosov, 1933]; West of Konda river, Mazurova, Kuchak, Ivanovka [Sitnikov, 2000, 2013; Red Data Book..., 2004; Tolstikov et al., 2013]; Kryukovskoe NM [Sitnikov, 2013]; Kondinskiye lakes NP [Ukhova, 2010; Kondinskiye..., 2012].

Distribution. Europe, European Russia, Siberia.

Lepturinae Latreille, 1802 Rhagiini

Rhagium (Megarhagium) mordax (DeGeer, 1775)

Rhagium mordax: Tyumen [Csíki, 1901]; «Tobolsk province» [Kiseleva, 1926]; Tobolsk [Plavilstshikov, 1936]; mount Neroika [Zinovyev, Olshvang, 2003]; Tobolsk, Missiya RS [Bukhkalo et al., 2011]; Kondinskiye lakes NP [Kondinskiye..., 2012].
Material. KHMAO: Surgutsky (Yugansky NR), Nizhnevartovsky (Agan River) districts. AS: Uvatsky (Uvat), Tobolsky (Vinokurova, Ovsyannikova), Yarkovsky (Mazurova), Nizhnetavdinsky (vicinity of Lake Kuchak, Lake Kultybayka), Tyumensky (Bogandinsky), Vikulovsky (Yushkova), Abatsky (Mayka), Isetsky (Maryino ushchelye NM), Aromashevsky (Novopetrovo) districts.

Distribution. Europe, European Russia, Siberia, Kazakhstan.

**Rhagium (Megarhagium) sycophanta (Schrank, 1781)

Rhagium sycophanta: Tobolsk [Plavilstshikov, 1915a; 1936].

Distribution. Europe, European Russia, ? West Siberia.

Remarks. In Western Siberia, including Tobolsk, it is known only by the old publications of N.N. Plavilstshikov. In modern studies on the territory of the Tyumenskaya oblast this species is not found, and its presence in the region is unlikely and requires confirmation.

\section{Rhagium (Rhagium) inquisitor Linnaeus, 1758}

Rhagium inquisitor: Tobolsk [Plavilstshikov, 1915a]; Oktyabrskoye [Korshunov, 1973]; Muravlenko [Zinovyev, Ryabitsev, 2000]; Tobolsk, Abalak, Missiya RS [Bukhkalo et al., 2011]; Kondinskiye lakes NP [Kondinskiye..., 2012].

Material. KHMAO: Surgutsky (Yugansky NR), Nizhnevartovsky (Agan River) districts. AS: Uvatsky (Kunyaksky reserve, Uvat), Tobolsky (Maslova, Vinokurova, Nadtsy), Nizhnetavdinsky (vicinity of Lake Kuchak), Tyumensky (vicinity of Lake Andreevskoe, Bogandinsky, Tyumen), Uporovsky (Staraya Shadrina), Armizonsky (Krashenevo) districts.

Distribution. Europe, European Russia, Caucasus, Siberia, Kazakhstan, Mongolia.

\section{Stenocorus (Stenocorus) meridianus} (Linnaeus, 1758)

Stenocorus meridianus: Pleshkovskie Uvaly NM, Urochishche Sogra NM [Lomakin et al., 2001; Red Data Book..., 2004; Stolbov et al., 2017]; Urochishche Cholpan NM [Krasutsky, Pekin, 2014]; Shashova [Galich, 2016].

Material. AS: Uporovsky distr., vicinity of Chernaya vill., 1.08.2017, 1 ex., V.I. Kapitonov.

Distribution. Europe, European Russia, Siberia, Kazakhstan.

\section{Pachyta lamed (Linnaeus, 1758)}

Pachyta lamed: Oktyabrskoye [Chugunov, 1917]; Konda river, Tobolsk [Kolosov, 1933]; Sibirskiye Uvaly NP [Zinovyev, Nesterkov, 2003]; «Tyumen oblast» [Danilevsky, 2014].

Material. YANAO: Yamalsky district (Yuribei river basin). KHMAO: Beryozovsky (Sosva, collection of IPAE), Surgutsky (Yugansky NR), Nizhnevartovsky (Pokur) districts. AS: Nizhnetavdinsky district (vicinity of Lake Kuchak).

Distribution. Europe, European Russia, Siberia, Far East, Mongolia, China, Korea, Japan.

\section{Pachyta quadrimaculata}

(Linnaeus, 1758)

Pachyta quadrimaculata: Armizonskoye [Striganova, Poryadina, 2005]; Abalak [Bukhkalo et al., 2011]; Kondinskiye lakes NP [Kondinskiye..., 2012].

Material. KHMAO: Surgutsky district (Yugansky NR). AS: Uvatsky (Missiya RS), Tobolsky (Tobolsk), Vagaysky (Kryukovskoe NM, Vagay), Yarkovsky (Mazurova), Nizhnetavdinsky (vicinity of Lake Kuchak, Lake Kultybayka), 
Tyumensky (Krivodanova, Golovina, Mullashi, Bogandinsky, Chervishevo, Kamenka), Isetsky (Maryino ushchelye NM, Bityuki), Zavodoukovsky (Komissarovsky reserve), Uporovsky (Uporovsky reserve), Armizonsky (Krashenevo) districts.

Distribution. Europe, European Russia, Siberia, Kazakhstan, Mongolia, China.

\section{Evodinellus (Evodinellus) borealis (Gyllenhal, 1827)}

Evodinellus borealis: «Tyumen region» [Danilevsky, 2014]. Material. KHMAO: Sovetsky distr., Sovetsky, 25.06.1982, T. Galasyeva (M.L. Danilevsky, pers. comm.); Nefteyugansky distr., Nefteyugansk, 11.07.1986, P.S. Sitnikov.

Distribution. North Europe, North European Russia, Siberia, Far East, Kazakhstan, Mongolia, China, Korea, Japan.

\section{Brachyta (Variobrachyta) variabilis (Gebler, 1817)}

Evodinus variabilis: Tobolsk [Plavilstshikov, 1915b]; «Tobolsk province» [Kiseleva, 1926].

Brachyta variabllis: "Tyumen oblast»[Danilevsky, 2014].

Distribution. European Russia, Siberia, Far East, Kazakhstan, Mongolia, China, Korea.

Remarks. Known only by the old collections (1929) of K.P. Samko (collection of Tobolsk Historical and Architectural Museum-Reserve) and literary mentions from the environs of Tobolsk.

\section{Brachyta (Brachyta) interrogationis (Linnaeus, 1758)}

Evodinus interrogationis: «Polar Ural»[Fridolin, 1935; Olshvang, 1980], Khadytayakha river [Olshvang, 1992]; Sibirskiye uvaly NP [Zinovyev, Malozemov, 2002]; mount Neroika, Shchekurya river [Zinovyev, Olshvang, 2003].

Brachyta interrogationis: Tobolsk [Csíki, 1901]; Rafaylovsky reserve [Obogrelov et al., 2002]; Tobolsk, Missiya RS [Bukhkalo et al., 2011]; Lake Shchuchye [Danilevsky, 2014].

Material. YANAO: Krasnoselkupsky district (Pyulky river) KHMAO: Beryozovsky (Pripolyarny), Nizhnevartovsky (Pokur) Surgutsky (Yugansky NR) districts. AS: Uvatsky (Uvat), Tobolsky (Verkhniye Aremzyany, Ovsyannikova), Nizhnetavdinsky (vicinity of Lake Kuchak), Yarkovsky (Mazurova), Tyumensky (Bogandinsky, Onokhino), Isetsky (Bityuki), Yalutorovsky (Lake Singul, Moshkarinsky reserve), Sladkovsky (Ostropyatovo, Tavolzhan) districts.

Distribution. Europe, European Russia, Caucasus, Siberia, Far East, Kazakhstan, Mongilia, Korea, China.

\section{Carilia virginea (Linnaeus, 1758)}

Gaurotes virginea: Tyumen, Khmelyova [Csíki, 1901]; Yalutorovsk [Kolosov, 1914]; Tobolsk, Vinokurova [Bukhkalo et al., 2011].

Carilia virginea: Tobolsk [Danilevsky, 2014].

Material. KHMAO: Surgutsky district (Yugansky NR, collection of IPAE). AS: Tobolsky (Abalak, Ovsyannikova), Nizhnetavdinsky (vicinity of Lake Kuchak, Velizhany), Yarkovsky (Mazurova), Tyumensky (Bogandinsky) districts.

Distribution. Europe, European Russia, Siberia, Far East, Kazakhstan, Mongilia, Korea, China.

\section{Euracmaeops marginatus}

(Fabricius, 1781)

Acmaeops marginata: Khmelyova [Csíki, 1901]; Shchuchya river [Shukhov, 1914]; Peregryobnoye, Oktyabrskoye [Kolosov, 1933].

Acmaeops marginatus: Kondinskiye lakes NP [Kondinskiye..., 2012].
Material. YANAO: Purovsky district (Gubkinsky) KHMAO: Nizhnevartovsky district (Sibirskiye uvaly NP, collection of IPAE). AS: Nizhnetavdinsky (vicinity of Lake Kuchak), Yarkovsky (Motushi) districts.

Distribution. Europe, European Russia, Siberia, Far East, Kazakhstan, Mongolia, China.

\section{Euracmaeops septentrionis}

(Thomson, 1866)

Acmaeops septentrionis: Salekhard [Cherepanov, 1970]; Venuyeuoyakha river [Ryabitsev, 1997].

Material. YANAO: Priuralsky (Kharp, collection of IPAE), Nadymsky (Nadymskoye gorodishche, collection of IPAE) districts. KHMAO: Surgutsky district (Yugansky NR).

Distribution. Europe, European Russia, Siberia and Russian Far East, Kazakhstan, Mongolia, China, Korea, Japan.

\section{Euracmaeops smaragdulus}

(Fabricius, 1793)

Acmaeops smaragdula: Salekhard, Peregryobnoye, Syoyakha [Kolosov, 1933]; Mount Payer [Fridolin, 1935]; «Polar Ural», «lower reaches of the Ob», Khadytayakha river [Olshvang, 1977, 1980, 1992].

Acmaeops smaragdulus: Sibirskiye uvaly NP [Zinovyev, Nesterkov, 2003]; Tobolsk [Bukhkalo et al., 2011].

Euracmaeops smaragdulus: Yamal [Danilevsky, 2014].

Material. YANAO: Purovsky (Novy Urengoy), Krasnoselkupsky (Krasnoselkup, Ratta) districts. KHMAO: Beryozovsky (Vogulka reserve, Beryozovo, Pripolyarnyy), Surgutsky (Yugansky NR) districts.

Distribution. North Europe, North European Russia, Siberia, Far East, Kazakhstan, Mongolia, China, Korea.

\section{Euracmaeops angusticollis} (Gebler, 1833)

Acmaeops angusticollis: Krasnoselkup [Sitnikov, 2000].

Distribution. Poland, Belarus, North European Russia, Siberia, Far East, Mongolia, China, Korea, Japan.

\section{Gnathacmaeops pratensis \\ (Laicharting, 1784)}

Acmaeops pratensis: Mount Payer [Fridolin, 1935]; Kharp, «lower reaches of the $\mathrm{Ob}$ », Khadytayakha river [Olshvang, 1977, 1980, 1992]; Malaya Sosva NR, Mazurova [Striganova, Poryadina, 2005]; Tobolsk, Vinokurova, Abalak [Bukhkalo et al., 2011]; Kondinskiye lakes NP [Kondinskiye..., 2012].

Material. YANAO: Priuralsky (Oktyabrsky, Salekhard, Labytnangi, Zelenyy Yar), Tazovsky (Tazovsky), Krasnoselkupsky (Upper Taz NR), Shuryshkarsky (Muzhi) districts. KHMAO: Beryozovsky (Beryozovo), Surgutsky (Yugansky NR), Nizhnevartovsky (Agan River, Pokur, Sibirskiye uvaly NP) districts. AS: Nizhnetavdinsky (Lake Kultybayka), Tyumensky (Borovsky) and Isetsky (Maryino ushchelye NM) districts.

Distribution. Europe, European Russia, Caucasus, Siberia, Far East, Central Asia, Mongolia, China, Korea.

\section{*Dinoptera collaris \\ (Linnaeus, 1758)}

Material. AS: Nizhnetavdinsky distr., vicinity of Lake Kuchak, 22.06.1996, 1 ex., A.N. Mokhratov, 29.06.1996, 1 ex., A.N. Mokhratov, 13.07.1997, 1 ex., A.N. Mokhratov, 13.06.2002, 1 ex., Samorokova, 6.07.2003, 2 ex., V.A. Stolbov, 10.07.2003, 1 ex., P. Vasilyev, 4.07.2009, 1 ex., Kozak, 15.07.2017, 1 ex., S.D. Sheykin; Lake Bayrak, 10.07.1994., 1 ex., P.S. Sitnikov; Lake Kultybayka, 9.07.1994, 1 ex., 
P.S. Sitnikov, 6.07.1995, 2 ex., P.S. Sitnikov, 3.07.1997, 1 ex., P.S. Sitnikov; Tyumensky distr., Embaevo vill., 14.07.2001, 1 ex., V.A. Abramov; Chervishevo vill., 4.07.1982, 1 ex. P.S. Sitnikov, 27.06.1987, 1 ex., P.S. Sitnikov; Levashi vill., 2.07.1985, 1 ex., P.S. Sitnikov; Zavodoukovsky distr., Gilyovo vill., 28.06.2006, 1 ex., V.A. Stolbov; Berdyuzhsky distr., Okunyovo vill., 10.07.2018, 2 ex., E.V. Sergeeva; Sladkovsky distr., Gulyay-Pole vill., 23.06.1990, 1 ex., P.S. Sitnikov; Ostropyatovo vill., 27.06.1990, 1 ex., P.S. Sitnikov; Sladkovo vill., 28.06.1990, 1 ex., P.S. Sitnikov.

Distribution. Europe, European Russia, Caucasus, West Siberia, Kazakhstan, Near East, China.

\section{Cortoderafemorata (Fabricius, 1787)}

Cortodera femorata: Tyumen [Sitnikov, 2000; Sitnikov et al., 2004; Red Data Book..., 2004]; Kuchak [Shapovalov, 2012; Danilevsky, 2014].

Material. AS: Nizhnetavdinsky distr., vicinity of Lake Kuchak, 16.06.2004, 1 ex., M.Yu. Lupinos; 01.07.2019, 1 ex., V.A. Stolbov.

Distribution. Europe, European Russia, West Siberia.

\section{Lepturini}

**Cornumutila lineata (Letzner, 1844)

Letzneria quadrivittata: Tobolsk [Kolosov, 1933].

Cornumutila quadrivittata: Sureya [Plavilstshikov, 1936].

Cornumutila lineata: Sureya [Lazarev, 2009; Danilevsky, 2014].

Distribution. Europe, European Russia, Siberia.

Remarks. In Tyumenskaya oblast is known only from old articles. Yu.M. Kolosov [1933] mentioned this species for Tobolsk as Letzneria quadrivittata Gebl., with the remark «the specimen somewhat different from the typical form». Based on the species range, we assume that this specimen belongs to the Cornumutila lineata (Letz.). This is evidenced by the later mention of this species [Plavilstshikov, 1936] for the Tobolsk district (Sureya), the specimen of which was later identified as Cornumutila lineata [Lazarev, 2009] (collection of ZIN).

\section{Nivellia sanguinosa (Gyllenhal, 1827)}

Nivellia sanguinosa: Tobolsk [Kolosov, 1933]; Tyumen [Cherepanov, 1979]; «Tyumen oblast» [Danilevsky, 2014].

Material. KHMAO: Beryozovsky (Pripolyarnyi), Surgutsky (Yugansky NR) districts. AS: Uvatsky district (Uvat).

Distribution. Europe, European Russia, Siberia and Russian Far East, Mongolia, China, Korea, Japan.

\section{**Nivellia extensa (Gebler, 1833)} 1980]

Nivellia extensa: Mount Payer [Fridolin, 1935; Olshvang,

Distribution. Finland, North European Russia, Siberia and Russian Far East, Mongolia, China, Korea, Japan.

Remarks. Known only by literature from Mount Payer (Shuryshkarsky distr., YANAO) without the exact geographical point. Finding this species in the region needs confirmation.

\section{Alosterna tabacicolor}

(DeGeer, 1775)

Alosterna tabacicolor: Tyumen [Csíki, 1901]; Khanty-Mansiysk [Zinovyev, 2005, 2008; Koltunov et al., 2009]; Durynina, Missiya RS [Bukhkalo et al., 2011]; Kondinskiye lakes NP [Kondinskiye..., 2012].
Material. KHMAO: Nefteyugansky (Nefteyugansk, Zapadno-Malobalykskoye oil field), Surgutsky (Yugansky NR) districts. AS: Nizhnetavdinsky (vicinity of Lake Kuchak, Lake Kultybayka), Yarkovsky (Mazurova), Tyumensky (vicinity of Lake Andreevskoe, Rechkina), Isetsky (Olkhovka river) districts.

Distribution. Europe, European Russia, Caucasus, Siberia and Russian Far East, Near East, Kazakhstan, Mongolia, China Korea, Japan.

\section{Pseudovadonia livida (Fabricius, 1777)}

Pseudovadonia livida: Missiya RS, Tobolsk, Abalak, Vinokurova [Bukhkalo et al., 2011].

Material. KHMAO: Surgutsky district (Yugansky NR). AS: Tobolsky (Ingair, Maslova), Nizhnetavdinsky (vicinity of Lake Kuchak), Yarkovsky (Dubrovnoe), Tyumensky (vicinity of Lake Andreevskoe), Uporovsky (Morevo, Pushkaryova), Berdyuzhsky (Berdyuzhye) districts.

Distribution. Europe, European Russia, Caucasus, Siberia, Near East, Kazakhstan, Central Asia.

\section{Stictoleptura (Aredolpona) rubra (Linnaeus, 1758)}

Leptura rubra: Tyumen [Csíki, 1901]; Oktyabrskoye [Korshunov, 1973].

Anoplodera rubra: Khanty-Mansiysk [Cherepanov, 1979].

Corymbia rubra: Samarovsky Chugas NP [Zinovyev, 2005, 2008; Koltunov et al., 2009].

Stictoleptura rubra: Tobolsk, Durynina, Abalak [Bukhkalo et al., 2011]; Khanty-Mansiysk [Danilevsky, 2014].

Material. AS: Uvatsky (Missiya RS), Tobolsky (Vinokurova, Maslova), Nizhnetavdinsky (vicinity of Lake Kuchak, Lake Kultybayka, Lake Bayrak), Yarkovsky (Mazurova), Tyumensky (vicinity of Lake Andreevskoe, Bogandinsky), Isetsky (Maryino ushchelye NM), Zavodoukovsky (Komissarovsky reserve), Uporovsky (Uporovsky reserve) districts.

Distribution. Europe, European Russia, Siberia, Kazakhstan.

\section{Stictoleptura (Variileptura) variicornis (Dalman, 1817)} 2002]

Anoplodera variicornis: Uvat, Nadtsy, Ingair [Sitnikov,

Corymbia variicornis: Uvat, Nadtsy, Ingair [Sitnikov et al., 2004; Red Data Book..., 2004]; Abalak [Alemasova, Galich, 2006]. 2011].

Stictoleptura variicornis: Tobolsk, Abalak [Bukhkalo et al.,

Material. AS: Uvatsky distr., vicinity of Missiya RS, 1314.08.2014, 4 ex., E.V. Sergeeva.

Distribution. North East Europe, European Russia, Siberia, Far East, Kazakhstan, Mongolia, China, Korea, Japan.

\section{Anastrangalia sanguinolenta (Linnaeus, 1760)}

Leptura sanguinolenta: Tyumen [Csíki, 1901]; Oktyabrskoye, Tobolsk [Kolosov, 1933]; Kondinskiye lakes NP [Kondinskiye..., 2012].

Leptura dubia: Oktyabrskoye, Strelovskoye [Chugunov, 1917].

Anastrangalia sanguinolenta: Samarovsky Chugas NP [Zinovyev, 2005, 2008; Koltunov et al., 2009].

Anoplodera sanguinolenta: Tobolsk [Bukhkalo et al., 2011].

Material. AS: Nizhnetavdinsky distr., vicinity of Lake Kuchak, 15.06.2004, 1 ex., A.L. Kulikova, 06.2008, 1 ex., V.A. Stolbov, 22.06.2010, 1 ex., V.A. Stolbov; Tyumensky distr., Krivodanova vill., 15.07.2004, 1 ex., V.A. Abramov; Isetsky distr., Maryino ushchelye NM, 28.07.2018, 1 ex., V.A. Stolbov. 
Distribution. Europe, European Russia, Caucasus, West Siberia, Kazakhstan.

\section{Anastrangalia reyi (Heyden, 1885)} 2009].

Anastrangalia reyi: Khanty-Mansiysk [Koltunov et al.,

Anoplodera reyi: Missiya RS, Vinokurova, Tobolsk, Abalak [Bukhkalo et al., 2011].

Material. KHMAO: Beryozovsky (Vogulka reserve, Beryozovo), Beloyarsky (Sorumsky reserve), Nefteyugansky (Nefteyugansk, Zapadno-Malobalykskoye oil field), Surgutsky (Yugansky NR) districts. AS: Tobolsky (Penya), Nizhnetavdinsky (vicinity of Lake Kuchak), Yarkovsky (Dubrovnoe, Motushi), Tyumensky (Borovsky, Tyumen), Isetsky (Olhovka river), Zavodoukovsky (Komissarovsky reserve), Abatsky (Konyovo), Sladkovsky (Gulyay-Pole) districts.

Distribution. Europe, European Russia, West Siberia, Kazakhstan.

\section{**Anastrangalia renardi (Gebler, 1848)}

Leptura renardi: Beryozovo [Kolosov, 1933].

Distribution. North European Russia, Siberia, Far East, Kazakhstan, Mongolia, China, Korea.

Remarks. Currently known only by old mention from Beryozovo (KHMAO). Finding this species in the region needs confirmation.

\section{Lepturobosca virens (Linnaeus, 1758)}

Leptura virens: Yalutorovsk [Kolosov, 1914]; «Tobolsk province» [Kiseleva, 1926]; Oktyabrskoye [Korshunov, 1973]; Noyabrsk [Zinovyev, Ryabitsev, 2000]; Sibirskiye uvaly NP [Zinovyev, Nesterkov, 2003]; Kondinskiye lakes NP [Kondinskiye..., 2012].

Lepturobosca virens: Samarovsky Chugas NP, Khanty-Mansiysk [Zinovyev, 2005, 2008; Koltunov et al., 2009]; Batovo [Gerasimov et al., 2015].

Anoplodera virens: Tobolsk, Abalak [Bukhkalo et al., 2011].

Material. YANAO: Krasnoselkupsky district (Upper Taz NR). KHMAO: Beryozovsky (Vogulka reserve, Pripolyarnyi), Beloyarsky (Sorumsky reserve), Oktyabrsky (Malyi Atlym), Sovetsky (Sovetsky), Nefteyugansky (Nefteyugansk, Pyt-Yakh), Surgutsky (Yugansky NR), Nizhnevartovsky (Pokur) districts. AS: Uvatsky (Kunyaksky reserve, Gornoslinkino, Missiya RS, Uvat), Tobolsky (Ingair, Vinokurova, Ovsyannikova, Maslova), Nizhnetavdinsky (vicinity of Lake Kuchak, Lake Kultybayka), Yarkovsky (Dubrovnoe, Mazurova), Tyumensky (Krivodanova, Bogandinsky, Chervishevo, Lake Lukashinskoye), Zavodoukovsky (Komissarovsky reserve), Omutinsky (Omutinsky reserve) districts.

Distribution. Europe, European Russia, Siberia, Far East, Kazakhstan, Mongolia, China.

\section{Judolia sexmaculata (Linnaeus, 1758)}

Leptura sexmaculata: Shchuchya river [Shukhov 1914; Samko, 1928b].

Judolia sexmaculata: Mount Payer, Lake Varchato [Fridolin, 1935]; Salekhard [Cherepanov, 1970]; «Polar Ural», «lower reaches of the Ob», Khadytayakha river [Olshvang, 1977, 1980, 1992; Zinovyev, Olshvang, 2003]; Samarovsky Chugas NP [Zinovyev, 2008; Koltunov et al., 2009]; Tobolsk [Bukhkalo et al., 2011]; Kondinskiye lakes NP [Kondinskiye..., 2012]; Yugansky NR [Shapovalov, 2012; Danilevsky, 2014]; Batovo [Gerasimov et al., 2015].

Material. KHMAO: Beryozovsky (Pripolyarnyi), Nefteyugansky (Nefteyugansk) districts. AS: Tobolsky (Abramova, Ingair, Verkhniye Aremzyany, Penya), Tyumensky (Uspensky reserve, vicinity of Lake Andreevskoe) districts.
Distribution. Europe, European Russia, West Siberia, Kazakhstan

Remarks. According to M.L. Danilevsky [2014], in Yugansky Nature Reserve area (Surgutsky distr., KHMAO) population of this species has a transitional character to the Judolia dentatofasciata (Mannerheim, 1852). Probably, the old literary mentions of $J$. sexmaculata from the territory of YANAO and from the north of KHMAO [Fridolin, 1936; Cherepanov, 1970; Olshvang, 1977, 1980, 1992; Zinovyev, Olshvang, 2003] should be attributed to J. dentatofasciata, cited for these territories in modern studies [Shapovalov, 2012; Danilevsky, 2014].

\section{**Judolia dentatofasciata (Mannerheim, 1852)}

Judolia dentatofasciata: Manya River, Kharp [Shapovalov, 2012]; Khadytayakha river, Shchuchya River [Danilevsky, 2014].

Distribution. North European Russia, Siberia, Far East, Mongolia, China, Korea, Japan.

\section{*Pachytodes erraticus (Dalman, 1817)}

Material. AS: Tyumensky distr., Kamenka vill., 1.07.1991, 1 ex., P.S. Sitnikov; Zavodoukovsky distr., Gilyovo vill., 14.07.2005, 1 ex., V.A. Stolbov; Komissarovsky reserve, 27.07.2003, 1 ex., A.V. Tolstikov; Omutinsky distr., 26.07.2003, 1 ex., A.V. Tolstikov; Ishimsky distr., Bolshoy Ostrov vill., 21.07.2000, 1 ex., P.S. Sitnikov; Armizonsky distr., Novoryamovo vill., 29.07.2017, 1 ex., V.A. Stolbov; Berdyuzhsky distr., Berdyuzhye vill., 7.08.2003, 1 ex., E.V. Sergeeva; Staroryamovo vill., 30.07.2017, 1 ex., V.A. Stolbov; Kazansky distr., Blagodatnoye vill., 24.07.2000, 1 ex., P.S. Sitnikov; Malye Yarki vill., 3.08.2014, 1 ex., S.A. Ivanov; Sladkovsky distr. Gulyay-Pole vill., 23.06.1990, 4 ex., P.S. Sitnikov; Kochkarnoye vill., 8.08.2018, 2 ex., P.S. Sitnikov.

Distribution. Europe, European Russia, Caucasus, Siberia, Kazakhstan, Near East.

\section{Oedecnema gebleri Ganglbauer, 1889}

Oedecnema dubia: Tyumen [Csíki, 1901].

Oedecnema gebleri: Tobolsk [Bukhkalo et al., 2011].

Material. KHMAO: Surgutsky district (Yugansky NR). AS: Tobolsky (Nadtsy), Nizhnetavdinsky (vicinity of Lake Kuchak), Tyumensky (Borovsky, Bogandinsky, Kamenka), Uporovsky (Emurtla, Shashova) districts.

Distribution. Ukraine, European Russia, Siberia, Far East, Kazakhstan, Mongolia, China, Korea, Japan.

\section{Leptura (Macroleptura) thoracica Creutzer, 1799}

Leptura thoracica: Lake Kultybayka, Dubrovnoe [Sitnikov, 2000]; Missiya RS [Bukhkalo et al., 2011].

Material. AS: Tobolsky (Tobolsk), Vagaysky (Kryukovskoe NM), Nizhnetavdinsky (vicinity of Lake Kuchak), Yarkovsky (Staroalexandrovka), Zavodoukovsky (Zavodoukovsk), Isetsky (Maryino ushchelye NM), Berdyuzhsky (Okunyovo, Polovinnoe) districts.

Distribution. Europe, European Russia, Caucasus, Siberia and Russian Far East, Kazakhstan, Mongolia, China, Korea, Japan.

\section{Leptura (Leptura) quadrifasciata Linnaeus, 1758}

Strangalia quadrifasciata: Yalutorovsk [Kolosov, 1914]; Strelovskoye [Chugunov, 1917]; Oktyabrskoye [Korshunov, 1973].

Leptura quadrifasciata: Samarovsky Chugas NP [Zinovyev, 2008; Koltunov et al., 2009]; Missiya RS, Tobolsk, Vinokurova [Bukhkalo et al., 2011]; Batovo [Gerasimov et al., 2015]. 
Material. YANAO: Krasnoselkupsky district (Ratta) KHMAO: Beloyarsky (Sorumsky reserve), Oktyabrsky (Peregryobnoye), Khanty-Mansiysky (Khanty-Mansiysk, Gornopravdinsk), Nefteyugansky (Nefteyugansk), Surgutsky (Yugansky NR), Nizhnevartovsky (Pokur) districts. AS: Tobolsky (Ingair, Verkhnive Aremzyany, Abalak, Maslova), Yarkovsky (Mazurova), Nizhnetavdinsky (vicinity of Lake Kuchak, Lake Kultybayka), Tyumensky (Krivodanova, Tyumen, Onokhino, vicinity of Lake Andreevskoe), Zavodoukovsky (Komissarovsky reserve, Gilyovo, Michurinsky), Vikulovsky (Yushkova), Omutinsky (Omutinsky reserve), Uporovsky (Pushkaryova), Armizonsky (Krashenyovo), Ishimsky (Ishim), Berdyuzhsky (Staroryamovo), Sladkovsky (Gulyay-Pole, Mayka, Tavolzhan) districts.

Distribution. Europe, European Russia, Caucasus, Siberia and Russian Far East, Kazakhstan, Mongolia, China, Korea, Japan.

\section{Leptura (Leptura) annularis Fabricius, 1801}

Leptura annularis: Tobolsk, Abalak [Bukhkalo et al., 2011].

Material. AS: Tobolsky (Ingair, Verkhniye Aremzyany, Ovsyannikova), Yarkovsky (Mazurova), Nizhnetavdinsky (vicinity of Lake Kuchak, Lake Bayrak, Lake Kultybayka, Velizhany), Tyumensky (Bogandinsky, Chervishevo), Zavodoukovsky (Komissarovsky reserve) districts.

Distribution. Europe, European Russia, Siberia, Far East, Kazakhstan, Mongolia, China, Korea, Japan.

\section{Leptura (Leptura) aethiops Poda, 1761}

Strangalia aethiops: Oktyabrskoye [Korshunov, 1973].

Leptura aethiops: Missiya RS, Vinokurova, Tobolsk [Bukhkalo et al., 2011].

Material. KHMAO: Beryozovsky (Pripolyarnyi, Volya river), Khanty-Mansiysky (Khanty-Mansiysk), Nefteyugansky (Salym, Nefteyugansk), Surgutsky (Yugansky NR), Nizhnevartovsky (Pokur) districts. AS: Uvatsky (Kunyaksky reserve), Tobolsky (Ingair, Ovsyannikova, Abalak, Penya), Nizhnetavdinsky (vicinity of Lake Kuchak), Tyumensky (Tyumen, Bogandinsky, Chervishevo, Levashi), Abatsky (Konyova) districts.

Distribution. Europe, European Russia, Caucasus, Siberia, Far East, Kazakhstan, Mongolia, China, Korea, Japan.

\section{Lepturalia nigripes (DeGeer, 1775)}

Strangalia nigripes: Yalutorovsk [Kolosov, 1914]; Strelovskoye [Chugunov, 1917]; Tyumen [Cherepanov, 1970].

Lepturalia nigripes: Missiya RS, Vinokurova, Tobolsk, Abalak [Bukhkalo et al., 2011]; Batovo [Gerasimov et al., 2015].

Material. YANAO: Krasnoselkupsky district (Ratta). KHMAO: Oktyabrsky (Peregryobnoye), Khanty-Mansiysky (Khanty-Mansiysk), Nefteyugansky (Nefteyugansk), Surgutsky (Yugansky NR), Nizhnevartovsky (Pokur) districts. AS: Tobolsky (Ingair), Nizhnetavdinsky (vicinity of Lake Kuchak, Lake Kultybayka), Yarkovsky (Dubrovnoe, Mazurova, Motushi), Tyumensky (vicinity of Lake Andreevskoe, Krivodanova, Bogandinsky, Lake Lukashinskoye, Lake Taraskul), Yalutorovsky (Lake Singul), Uporovsky (Pushkaryova, Shashova), Omutinsky (Omutinsky reserve), Abatsky (Mayka), Ishimsky (Ishim), Sladkovsky (Sladkovo, Travnoye, Ostropyatovo, Gulyay-Pole, Tavolzhan) districts.

Distribution. Europe, European Russia, Siberia, Far East, Kazakhstan, Mongolia, China, Korea.

Remarks. In the collections there are specimens, corresponding by colouration of both the western ( $L$. nigripes nigripes Deg.) and eastern (L. nigripes rufipennis (Blessig, 1873)) subspecies. In the territory of Western Siberia, there is a zone of contact between two subspecies [Danilevsky, 2014], herewith according to the materials of the collections studied, more than $70 \%$ of the specimens belong to the nominative subspecies.

\section{Strangalia attenuata (Linnaeus, 1758)}

Leptura attenuata: Chernaya Rechka [Redikortsev, 1908]; Yalutorovsk [Kolosov, 1914].

Strangalia attenuata: Tobolsk [Bukhkalo et al., 2011].

Material. AS: Nizhnetavdinsky (vicinity of Lake Kuchak, Lake Kultybayka), Yarkovsky (Mazurova), Tyumensky (vicinity of Lake Andreevskoe, Bogandinsky, Kamenka), Isetsky (Maryino ushchelye NM), Uporovsky (Uporovsky reserve), Armizonsky (Krashenyovo) districts.

Distribution. Europe, European Russia, Caucasus, Siberia, Far East, Kazakhstan, Near East, Mongolia, China, Korea, Japan.

\section{Stenurella (Stenurella) melanura (Linnaeus, 1758)}

Strangalia melanura: Oktyabrskoye [Korshunov, 1973].

Leptura melanura: Vinokurova, Tobolsk, Abalak [Bukhkalo et al., 2011].

Material. AS: Yarkovsky (Mazurova), Nizhnetavdinsky (vicinity of Lake Kuchak, Lake Kultybayka, Lake Bayrak), Tyumensky (Tyumen, Krivodanova, Bogandinsky, Chervishevo, vicinity of Lake Andreevskoe), Zavodoukovsky (Komissarovsky reserve), Uporovsky (Uporovsky reserve) districts.

Distribution. Europe, European Russia, Caucasus, Siberia, Far East, Kazakhstan, Near East, Mongolia, Japan.

\section{*Stenurella (Priscostenurella) bifasciata} (Müller, 1776)

Material. AS: Nizhnetavdinsky distr., vicinity of Lake Kuchak, 10.07.1997, 1 ex., A.N. Mokhratov, 25.06.2005, 1 ex., V.A. Abramov, 2.07.2012, 3 ex., V.A. Stolbov, 15.07.2017, 1 ex., S.D. Sheykin; Lake Kultybayka, 22.07.1993, 1 ex., P.S. Sitnikov; Velizhany vill., 25.06.1997, 2 ex., P.S. Sitnikov; Yarkovsky distr., Dubrovnoe vill., 8.07.2001, 1 ex., D.I. Sozinov; Mazurova vill., 12.07.1979, 1 ex., P.S. Sitnikov; Tyumensky distr., Tyumen, 20.07.2014, 1 ex., S.A. Ivanov; Chervishevo vill., 16.06.1997, 1 ex., P.S. Sitnikov; vicinity of Lake Andreevskoe, 21.07.1973, 1 ex., P.S. Sitnikov, 23.07.1992, 2 ex., A.V Tolstikov; Lake Lukashinskoye, 28.06.1987, 1 ex., P.S. Sitnikov; Zavodoukovsky distr., Gilyovo vill., 25.07.2005, 1 ex., V.A. Stolbov; Sladkovsky distr, Mayka vill., 29.06.1990, 1 ex., P.S. Sitnikov; Usovo vill., 9.08.2018, 2 ex., P.S. Sitnikov.

Distribution. Europe, European Russia, Caucasus, Siberia, Kazakhstan, Near East.

\section{Necydalinae Latreille, 1825 Necydalini}

Necydalis (Necydalis) major (Linnaeus, 1758)

Necydalis major: Denshchikova, Kosheleva [Chugunov, 1917]; Tobolsk [Samko, 1928à]; Oktyabrskoye [Korshunov, 1973]; vicinity of Lake Andreevskoe, Lake Kultybayka, Lake Bayrak, Dubrovnoe, Singulskiy forest NM [Sitnikov, 2000; Sitnikov et al., 2004; Red Data Book..., 2004]; Tobolsk, Degteryovo [Alemosova, Galich, 2006; Bukhkalo et al., 2011].

Material. KHMAO: Beryozovsky (Vogulka reserve), Surgutsky (Yugansky NR) districts. AS: Uvatsky (Missiya RS), Vagaysky (Kryukovskoe NM), Nizhnetavdinsky (vicinity of Lake Kuchak), Yarkovsky (Motushi), Tyumensky (Tyumen) districts.

Distribution. Europe, European Russia, Siberia, Far East, Kazakhstan, Near East, Mongolia, China, Korea, Japan.

\section{Spondylidinae Audinet-Serville, 1832 Asemini}

Asemum striatum (Linnaeus, 1758)

Asemum striatum: Khmelyova [Csíki, 1901]; Plavilstshikov, 1930; Oktyabrskoye [Korshunov, 1973]; Kharp, «lower reaches of the Ob» [Olshvang, 1977, 1980]; Missiya RS [Bukh- 
kalo et al., 2011]; Kondinskiye lakes NP [Kondinskiye..., 2012].

Material. KHMAO: Beryozovsky (Vogulka reserve), Surgutsky (Yugansky NR) districts. AS: Tobolsky (Tobolsk), Nizhnetavdinsky (vicinity of Lake Kuchak), Yarkovsky (Mazurova), Tyumensky (Borovsky, Tyumen, Onokhino) districts.

Distribution. Europe, European Russia, Caucasus, Siberia, Far East, Near East, Kazakhstan, Central Asia, Mongolia, China, Korea, Japan.

\section{Arhopalus (Arhopalus) rusticus (Linnaeus, 1758)}

Criocephalus rusticus: Beryozovo [Kiseleva, 1926]; Tyumen oblast [Cherepanov, 1970]; Oktyabrskoye [Korshunov, 1973]; Kondinskiye lakes NP [Kondinskiye..., 2012].

Arhopalus rusticus: Sibirskiye uvaly NP [Zinovyev, Nesterkov, 2003]; Missiya RS [Bukhkalo et al., 2011].

Material. YANAO: Krasnoselkupsky district (Krasnoselkup, Ratta). KHMAO: Beryozovsky (Vogulka reserve), KhantyMansiysky (Khanty-Mansiysk), Surgutsky (Yugansky NR) districts. AS: Tobolsky (Tobolsk, Penya), Nizhnetavdinsky (vicinity of Lake Kuchak, Lake Kultybayka), Yarkovsky (Dubrovnoe, Mazurova), Tyumensky (Tyumen, Embayevo, Krivodanova, vicinity of Lake Andreevskoe), Uporovsky (Emurtla) districts.

Distribution. North Africa, Europe, European Russia, Caucasus, Siberia, Far East, Near East, Kazakhstan, Central Asia, Mongolia, China, Korea, Japan.

\section{Tetropium castaneum \\ (Linnaeus, 1758)}

Tetropium castaneum: Tobolsk [Kolosov, 1933].

Tetropium gracilicorne: Tobolsk [Bukhkalo et al., 2011].

Material. YANAO: Krasnoselkupsky district (Krasnoselkup). KHMAO: Beloyarsky (Sorumsky reserve), Nefteyugansky (Zapadno-Malobalykskoye oil field), Surgutsky (Yugansky NR) districts.

Distribution. Europe, European Russia, Caucasus, Siberia, Far East, Near East, Kazakhstan, Mongolia, China, Korea, Japan.

\section{*Tetropium gracilicorne Reitter, 1889}

Material. YANAO, Krasnoselkupsky distr., environs of Krasnoselkup vill., 12.07.1989, 4 ex., P.S. Sitnikov (collection of MK).

Distribution. North European Russia, Siberia, Far East, Kazakhstan, Mongolia, China, Korea.

\section{**Tetropium aquilonium Plavilstshikov, 1940}

Tetropium aquilonium: Kushevat [Plavilstshikov, 1940; Danilevsky, 2014]; Khadytayakha river [Olshvang, 1980]; Venuyeuoyaha river [Ryabitsev, 1997].

Distribution. Sweden, Finland, North European Russia, West Siberia

\section{Spondylidini}

*Spondylis buprestoides (Linnaeus, 1758)

Material. AS: Nizhnetavdinsky distr., vicinity of Lake Kuchak, 29.06.1995, 1 ex., L.D. Golosova, 9.07.1996, 1 ex. A.N. Mokhratov, 10.07.1997, 1 ex., A.N. Mokhratov, 15.07.1997, 1 ex., A.N. Mokhratov; Lake Kultybayka, 9.07.1995, 1 ex., P.S. Sitnikov, 7.07.1996, 1 ex., P.S. Sitnikov; Yarkovsky distr., Dubrovnoe vill., 7.07.2001, 1 ex., V.A. Abramov, 1.07.2001, 3 ex., V.A. Abramov; Mazurova vill., 25.06.1989, 1 ex., P.S. Sitnikov; Tyumensky distr., Krivodanova vill., 1.07.2002, 1 ex., V.A. Abramov, 26.07.2003, 1 ex.,
V.A. Abramov, 11.07.2004, 6 ex., V.A. Abramov; vicinity of Lake Andreevskoe, 23.07.1988, 1 ex., P.S. Sitnikov, 20.07.2003, 2 ex., V.A. Stolbov; Onokhino vill., 29.06.2016, 1 ex., P.S. Sitnikov; Uporovsky distr., Uporovsky reserve, 29.07.2003, 6 ex., A.V. Tolstikov.

Distribution. Europe, European Russia, Caucasus, Siberia, Far East, Near East, Kazakhstan, Central Asia, Mongolia, China, Korea, Japan.

Cerambycinae Latreille, 1802

Purpuricenini

Purpuricenus globulicollis Mulsant, 1839

Purpuricenus tsherepanovae: Lake Taraskul, Kuchak [Sitnikov, 1998, 2000].

Purpuricenus kaehleri: Lake Taraskul, Kuchak [Sitnikov, 1998, 2000]; Lake Taraskul, Kuchak, Tavolzhan [Red Data Book ..., 2004]; Tavolzhan [Tolstikov et al., 2013].

Purpuricenus globulicollis: Uspenka [Danilevsky et al., 2007]; Kuchak [Stolbov et al., 2017].

Distribution. Europe, European Russia, West Siberia, Kazakhstan.

\section{Callichromatini Aromia moschata (Linnaeus, 1758)}

Aromia moschata: Yalutorovsk [Kolosov, 1928]; Sovetsky, Ingair, Tobolsk, Tyumen, Chervishevo [Sitnikov, 2000, 2002; Red Data Book ..., 2004], Maryino ushchelye NM [Obogrelov et al., 2002; Red Data Book ..., 2004]; Abalak reserve, Kryukovskoe NM, Pushkaryova [Sitnikov, 2013; Tolstikov et al., 2013]; urochishche Cholpan NM [Krasutsky, Pekin, 2014]; Kuchak [Tolstikov et al., 2013; Galich, 2016; Stolbov et al., 2017].

Material. AS: Tyumensky distr., vicinity of Lake Andreevskoe, 24.06.2007, 1 ex., S.D. Sheykin; Isetsky distr., Maryino ushchelye NM, 28.07.2018, 1 ex., V.A. Stolbov.

Distribution. Europe, European Russia, Caucasus, Siberia, Kazakhstan, Mongolia.

Obriini

Obrium cantharinum (Linnaeus, 1767)

Obrium cantharinum: Tobolsk, Tyumen, Lake Kultybayka [Sitnikov, 2000].

Obrium brunneum: Tobolsk [Bukhkalo et al., 2011].

Material. AS: Yarkovsky (Dubrovnoe, Mazurova), Tyumensky (Embaevo, vicinity of Lake Andreevskoe), Yalutorovsky (Sosnovka) districts.

Distribution. Europe, European Russia, Caucasus, Siberia, Far East, Kazakhstan, Mongolia, Northwest China.

\section{Molorchini \\ Molorchus (Caenoptera) minor (Linnaeus, 1758)}

Molorchus minor: Tobolsk [Bukhkalo et al., 2011].

Material. KHMAO: Surgutsky distr., Yugansky NR, Nyogusyakh river, science station Koganchino-2, 22.06.1997, 1 ex., A.N. Mokhratov. AS: Tobolsk, on Padus avium, 15.06.2013, 2 ex., 5.06.2015, 1 ex., 20.05.2017, 2 ex., E.V. Sergeeva.

Distribution. Europe, European Russia, Caucasus, Siberia, Far East, Kazakhstan, Mongolia, China, Korea.

\section{Molorchus (Molorchus) umbellatarus (Schreber, 1759)}

Molorchus umbellatarus: Tobolsk [Bukhkalo et al., 2011]. Distribution. Europe, European Russia, Caucasus, Turkmenistan.

Remarks. The only mention of this species for the territory of Western Siberia. 
Hylotrupini

**Hylotrupes bajulus (Linnaeus, 1758) 2009].

Hylotrupes bajulus: Khanty-Mansiysk [Koltunov et al.,

Distribution. North Africa, Europe, European Russia, Caucasus, West Siberia, China.

Remarks. Finding this species in the region needs confirmation.

\section{Callidiini}

*Pronocera sibirica (Gebler, 1848)

Material. KHMAO, Surgutsky distr., Yugansky NR, Nyogusyakh river, science station Koganchino-2, 9.07.1997, 1 ex., A.N. Mokhratov.

Distribution. Siberia, Far East, Mongolia, Korea, China.

*Semanotus undatus (Linnaeus, 1758)

Material. AS: Tobolsk, in a new (two-year-old) pine log house, 12.04.2016, 1 ex., E.V. Sergeeva.

Distribution. Europe, European Russia, Siberia and Russian Far East, Mongolia, China, Japan.

\section{Callidium (Palaeocallidium) coriaceum Paykull, 1800}

Callidium coriaceum: Kharp [Olshvang, 1977], "lower reaches of the $\mathrm{Ob}$ river» [Olshvang, 1980].

Material. KHMAO: Beloyarsky distr., Sorumsky reserve, 16.07.2007, 2 ex., V.A. Stolbov.

Distribution. Europe, European Russia, Siberia and Russian Far East, Mongolia, China, Japan.

Callidium (Callidium) violaceum (Linnaeus, 1758)

Callidium violaceum: Oktyabrskoye [Korshunov, 1973]; Samarovsky Chugas NP, Pyt-Yakh, Yugansky NR [Zinovyev, 2008; Koltunov et al., 2009]; Missiya RS, Tobolsk, Vinokurova, Abalak [Bukhkalo et al., 2011]; Kondinskiye lakes NP [Kondinskiye..., 2012].

Material. AS: Uvatsky (Demyanskoye), Tobolsky (Maslova, Nadtsy), Nizhnetavdinsky (vicinity of Lake Kuchak), Yarkovsky (Motushi), Tyumensky (Tyumen) districts.

Distribution. Europe, European Russia, Caucasus, Siberia, Far East, Kazakhstan, Mongolia, China, Korea, Japan.

\section{**Callidium (Callodostola) aeneum} (DeGeer, 1775)

Callidium aeneum: Beryozovo [Kolosov, 1933]; Salekhard [Cherepanov, 1981]; Noyabrsk, Muravlenko [Zinovyev, Ryabitsev, 2000].

Distribution. Europe, European Russia, Siberia, Far East, Kazakhstan, Mongolia, China, Japan.

\section{Clytini}

Echinocerus floralis (Pallas, 1773)

Echinocerus floralis: Soloboevo, Sinitsinskiy bor, Afonkino NM [Sitnikov, 2010]; Bityuki [Sitnikov et al., 2013].

Distribution. Europe, European Russia, Caucasus, Siberia, Kazakhstan, Central Asia.

\section{Chlorophorus (Immaculatus) herbstii (Brahm, 1790)}

Chlorophorus herbstii: Kuchak [Sitnikov, 2000; Red Data Book..., 2004]; Kryukovskoe NM [Sitnikov 2013].

Material. AS: Tobolsk, 23.05.2015, 1 ex., E.V. Sergeeva; Nizhnetavdinsky distr., vicinity of Lake Kuchak, 02.07.2019, 2 ex., V.A. Stolbov.
Distribution. Europe, European Russia, Siberia, Kazakhstan.

\section{Rhaphuma gracilipes Faldermann, 1835}

Rhaphuma gracilipes: Tobolsk [Bukhkalo et al., 2011].

Material. AS: Nizhnetavdinsky (vicinity of Lake Kuchak, Guzeneevsky reserve), Yarkovsky (Mazurova), Tyumensky (Tyumen, vicinity of Lake Andreevskoe), Uporovsky (Pushkaryova, Uporovo), Berdyuzhsky (Berdyuzhye, Okunyovo), Sladkovsky (Ostropyatovo) districts.

Distribution. Europe, European Russia, Siberia, Far East, Kazakhstan, Mongolia, China, Korea.

\section{Xylotrechus (Xylotrechus) arvicola (Olivier, 1795)}

Xylotrechus arvicola: Dubrovnoe [Sitnikov, 2000; Red Data Book ..., 2004; Sitnikov et al., 2013].

Distribution. North Africa, Europe, European Russia, Caucasus, West Siberia (Tyumen region), Kazakhstan, Iran.

Xylotrechus (Xylotrechus) capricornus (Gebler, 1830)

Xylotrechus capricornus: «Tobolsk Okrug» [Plavilstshikov, 1940]; Tyumen, Verkhny bor, Kuchak [Sitnikov, 2000; Red Data Book ..., 2004; Sitnikov et al., 2013].

Distribution. Europe, European Russia, West Siberia, Kazakhstan.

Xylotrechus (Rusticoclytus) rusticus (Linnaeus, 1758)

Xylotrechus rusticus: Sukhorukovo [Kolosov, 1933]; Oktyabrskoye [Korshunov, 1973]; Khanty-Mansiysk [Zinovyev, 2005, 2008; Koltunov et al., 2009]; Missiya RS [Bukhkalo et al., 2011]; Batovo [Gerasimov et al., 2015].

Material. KHMAO: Nefteyugansky (Nefteyugansk), Surgutsky (Yugansky NR) districts. AS: Tobolsky (Tobolsk, Nadtsy), Yarkovsky (Motushi), Nizhnetavdinsky (vicinity of Lake Kuchak, Lake Kultybayka), Tyumensky (Tyumen, Bogandinsky, Uspenka), Zavodoukovsky (Gilyovo), Sladkovsky (Sladkovo) districts.

Distribution. North Africa, Europe, European Russia, Caucasus, Siberia, Far East, Near East, Kazakhstan, Central Asia, Mongolia, China, Korea, Japan.

\section{Xylotrechus (Rusticoclytus) pantherinus (Savenius, 1825)}

Xylotrechus pantherinus: Lake Kultybayka [Sitnikov, 2000; Red Data Book..., 2004].

Distribution. Europe, European Russia, Siberia, Far East, Kazakhstan, Mongolia, China, Korea.

\section{Cyrtoclytus capra (Germar, 1824)}

Cyrtoclytus capra: Yalutorovsk [Kolosov, 1914]; Missiya RS, Vinokurova [Bukhkalo et al., 2011].

Material. AS: Tobolsky (Verkhniye Aremzyany, Penya), Yarkovsky (Mazurova), Nizhnetavdinsky (vicinity of Lake Kuchak, Shapkul, Lake Kultybayka), Tyumensky (Tyumen, Yembaevo, Bogandinsky, Gorkovka, Kulakovo), Isetsky (Maryino ushchelye NM), Zavodoukovsky (Michurinsky), Uporovsky (Uporovo), Berdyuzhsky (Okunyovo), Sladkovsky (Mayka, Travnoye, Ostropyatovo, Gulyay-Pole, Tavolzhan) districts.

Distribution. Europe, European Russia, Caucasus, Siberia, Far East, Kazakhstan, Mongolia, China, Korea.

\section{Clytus (Clytus) arietoides Reitter, 1899}

Clytus arietoides: Vinokurova [Bukhkalo et al., 2011].

Material. YANAO: Priuralsky distr., Khadytayakha river, 1.07.1973, V.N. Olshvang (collection of IPAE). AS: Tobolsk, 10.07.2015, 10.06.2017, 2 ex., E.V. Sergeeva. 
Distribution. European Russia, Siberia, Far East, Kazakhstan, Mongolia, China, Korea, Japan.

\section{Lamiinae Latreille, 1825 \\ Mesosini}

Mesosa (Mesosa) myops (Dalman, 1817)

Mesosa myops: Missiya RS [Bukhkalo et al., 2011].

Material. AS: Tobolsky (Tobolsk, Verkhniye Aremzyany), Vagaysky (Kryukovskoe NM), Nizhnetavdinsky (vicinity of Lake Kuchak, Lake Kultybayka), Yarkovsky (Mazurova), Tyumensky (Tyumen, Bogandinsky), Zavodoukovsky (Gilyovo) districts.

Distribution. Europe, European Russia, Siberia, Far East, Kazakhstan, Mongolia, Korea, China.

\section{Monochamini}

*Monochamus (Monochamus) impluviatus (Motschulsky, 1859)

Material. KHMAO, Nizhnevartovsky distr., upper course of the river Kolekyogan, found on Pinus sibirica, 12.08.1985, 1 ex., P.S. Sitnikov (collection of MK).

Distribution. European Russia, Siberia, Far East, Mongolia, Korea, Northeast China.

Monochamus (Monochamus) saltuarius (Gebler, 1830)

Monochamus saltuarius: Kondinskiye lakes NP [Kondinskiye..., 2012].

Material. KHMAO: Nizhnevartovsky distr., upper course of the river Kolekyogan, found on Pinus sibirica, 12.08.1985, 2 ex., P.S. Sitnikov (collection of MK); Novomolodezhnoye oil field, 17.07.1993, 1 ex., T.A. Sharapova. AS: Tobolsky distr., Maslova vill., spruce-fir-pine forest, on freshly cut pine logs, 23.08.2017, 1 ex., E.V. Sergeeva.

Distribution. Europe, European Russia, Siberia, Far East, Kazakhstan, Mongolia, China, Korea, Japan.

\section{Monochamus (Monochamus) galloprovincialis (Olivier, 1795)}

Monochamus galloprovincialis: Yalutorovsk [Kolosov, 1914]; Beryozovo [Kiseleva, 1926]; Khanty-Mansiysk [Cherepanov, 1970]; Oktyabrskoye [Korshunov, 1973]; Missiya RS [Bukhkalo et al., 2011]; Kondinskiye lakes NP [Kondinskiye..., 2012].

Material. YANAO: Krasnoselkupsky (Ratta, collection of IPAE), Shuryshkarsky (Lesmiegan river, collection of IPAE) districts. KHMAO: Surgutsky district (Yugansky NR, Pilugina, Ugut). AS: Uvatsky (Kunyaksky reserve, Gornoslinkino), Tobolsky (Tobolsk, Vinokurova, Verkhniye Aremzyany, Ovsyannikova, Abalak, Maslova, Nadtsy), Vagaysky (Kryukovskoe NM), Yarkovsky (Dubrovnoe, Mazurova), Nizhnetavdinsky (vicinity of Lake Kuchak, Lake Kultybayka), Tyumensky (Tyumen, Krivodanova, Bogandinsky, vicinity of Lake Andreevskoe), Yalutorovsky (), Isetsky (Maryino ushchelye NM), Uporovsky (Uporovsky reserve) districts.

Distribution. North Africa, Europe, European Russia, Caucasus, Siberia, Kazakhstan, Kyrgyzstan, Near East, Mongolia, China.

\section{Monochamus (Monochamus) urussovii}

(Fischer von Waldheim, 1805)

Monochamus rosenmuelleri: Tobolsk [Jacobson, 1910].

Monochamus quadrimaculatus: Tyumen [Csíki, 1901]; Tobolsk [Jacobson, 1910].

Monochamus urussovi: Oktyabrskoye [Korshunov, 1973]; Sibirskiye uvaly NP [Zinovyev, Nesterkov, 2003]; KhantyMansiysk [Zinovyev, 2005, 2008; Koltunov et al., 2009]; Missiya RS, Tobolsk [Bukhkalo et al., 2011].
Material. YANAO: Shuryshkarsky (Ust-Voykary), Krasnoselkupsky (Ratta), Purovsky (Novy Urengoy) districts. KHMAO: Beryozovsky (Vogulka reserve), Beloyarsky (Sorumsky reserve), Nefteyugansky (Zapadno-Malobalykskoye oil field), Surgutsky (Yugansky NR, Pilugina), Nizhnevartovsky (upper course of the river Kolekyogan) districts. AS: Tobolsky (Vinokurova, Ovsyannikova, Abalak, Maslova), Vagaysky (Kryukovskoe NM), Yarkovsky (Mazurova), Nizhnetavdinsky (vicinity of Lake Kuchak, Lake Kultybayka), Tyumensky (Bogandinsky), Zavodoukovsky (Gilyovo) districts.

Distribution. East Europe, European Russia, Caucasus, Siberia, Far East, Kazakhstan, Mongolia, Korea, China, Japan.

\section{Monochamus (Monochamus) sutor (Linnaeus, 1758)}

Monochamus sutor: Khmeleva [Csíki, 1901]; Salekhard, Bolshoy Atlym [Jacobson, 1910]; Labytnangi [Olshvang, 1980]; Oktyabrskoye [Chugunov, 1917; Korshunov, 1973; Cherepanov, 1983]; Noyabrsk, Muravlenko [Zinovyev, Ryabitsev, 2000]; Sibirskiye uvaly NP [Zinovyev, Nesterkov, 2003]; Missiya RS, Tobolsk [Bukhkalo et al., 2011]; Kondinskiye lakes NP [Kondinskiye..., 2012].

Material. YANAO: Yamalsky (Kharasavey, Yuribei river basin), Shuryshkarsky (Ust-Voykary), Tazovsky (vicinity of Antipayuta, Tazovsky), Krasnoselkupsky (Ratta, Tolka), Purovsky (Novy Urengoy) districts. KHMAO: Beloyarsky (Sorumsky reserve), Sovetsky (Sovetsky), Nefteyugansky (Nefteyugansk), Surgutsky (Yugansky NR, Pilugino), Nizhnevartovsky (Bolshetarkhovo, upper course of the river Kolekyogan) districts. AS: Uvatsky (Kunyaksky reserve, Gornoslinkino, Gerasimovka, Uvat, Demyanskoe), Tobolsky (Vinokurova, Ovsyannikova, Abalak, Maslova, Nadtsy), Vagaysky (Kryukovskoe NM), Yarkovsky (Dubrovnoe, Mazurova), Nizhnetavdinsky (vicinity of Lake Kuchak, Lake Kultybayka), Tyumensky (Tyumen, Krivodanova, Bogandinsky, Chervishevo, Lake Lukashinskoye), Zavodoukovsky (Gilyovo) districts.

Distribution. Europe, European Russia, Caucasus, Siberia, Far East, Kazakhstan, Mongolia, Korea, China, Japan.

Lamiini

\section{*Lamia textor (Linnaeus, 1758)}

Material. AS: Yarkovsky distr., Mazurova vill., 13.07.1975, 1 ex., P.S. Sitnikov; Nizhnetavdinsky distr., vicinity of Lake Kuchak, 23.06.1996, 2 ex., A.N. Mokhratov, 3.07.2000, 1 ex., unknown collector; 24.06.2002., 1 ex., D. Novak, 18.06.2005, 1 ex., S.D. Sheykin, 6.2012, 2 ex., unknown collector; 6.2013, 1 ex., R. Mamedov, 7.2013, 1 ex., Yesakov, 9.07.15, 1 ex., M.O. Filimonova, 22.06.2018, 6 ex., V.A. Stolbov; Tyumensky distr., Chervishevo vill., 5.1975, 2 ex., P.S. Sitnikov.

Distribution. Europe, European Russia, Caucasus, Siberia, Far East, Near East, Kazakhstan, Mongolia, Korea, China, Japan.

\section{Apodasyini}

Anaesthetis testacea (Fabricius, 1781)

Anaesthetis testacea: Durynina [Bukhkalo et al., 2011].

Material. AS: Zavodoukovsky distr., vicinity of Markovo vill., dry meadow near the birch forest, 27.06.2006, 1 ex., V.A. Stolbov.

Distribution. Europe, European Russia, Caucasus, West Siberia (Tyumen region), Near East, Kazakhstan.

\section{Pogonocherini \\ Pogonocherus (Pityphilus) fasciculatus}

(DeGeer, 1775)

Pogonocherus fasciculatus: Oktyabrskoye [Korshunov, 1973]; Tobolsk, Abramova [Bukhkalo et al., 2011]; Kondinskiye lakes NP [Kondinskiye..., 2012]. 
Material. YANAO: Priuralsky district (Salekhard, Kharp) KHMAO: Beloyarsky (Numto NP), Surgutsky (Yugansky NR) districts. AS: Tobolsky (Abalak), Nizhnetavdinsky (vicinity of Lake Kuchak, Lake Kultybayka), Tyumensky (Tyumen, Borovsky, Krivodanova, vicinity of Lake Andreevskoe), Aromashevsky (Alabuga reserve), Armizonsky (Poloe) districts.

Distribution. Europe, European Russia, Caucasus, Siberia, Far East, Near East, Kazakhstan, Mongolia, Korea, China, Japan.

Acanthoderini

$$
\text { Aegomorphus clavipes (Schrank, 1781) }
$$

Acanthoderes clavipes: Tobolsk [Bukhkalo et al., 2011]

Material. AS: Uvatsky (Missiya RS), Tobolsky (Verkhniye Aremzyany, Ingair), Nizhnetavdinsky (vicinity of Lake Kuchak, Lake Kultybayka), Yarkovsky (Mazurova, Motushi), Tyumensky (Mullashi, Bogandinsky, Chervishevo), Uporovsky (Uporovo), Armizonsky (Krashenyovo) districts.

Distribution. North Africa, Europe, European Russia, Caucasus, Siberia, Far East, Near East, Kazakhstan, Mongolia, Korea, China, Japan.

\section{Acanthocinini \\ Acanthocinus (Acanthocinus) griseus}

(Fabricius, 1793)

Acanthocinus griseus: Tyumen [Csíki, 1901].

Acanthocinus carinulatus: Missiya RS, Abalak [Bukhkalo et al., 2011].

Material. KHMAO: Nefteyugansky (Pyt-Yakh, collection of IPAE), Surgutsky (Yugansky NR, collection of IPAE) districts. AS: Tobolsk, Vagaysky (Ashlyk), Nizhnetavdinsky (Lake Kultybayka), Yarkovsky (Motushi), Tyumensky (Kamenka, Lake Lukashinskoye), Zavodoukovsky (Gilyovo), Sladkovsky (Vlasovo) districts.

Distribution. Europe, European Russia, Caucasus, Siberia, Kazakhstan.

\section{**Acanthocinus (Acanthocinus) carinulatus (Gebler, 1833)}

Acanthocinus carinulatus: Kondinskiye lakes NP [Kondinskiye..., 2012].

Distribution. Siberia, Mongolia, China.

Remarks. Finding this species in the region needs confirmation.

\section{Acanthocinus (Acanthocinus) aedilis} (Linnaeus, 1758)

Acanthocinus aedilis: former Voroninskiye Yurty [Chugunov, 1917]; Oktyabrskoye [Korshunov, 1973]; Noyabrsk, Muravlenko [Zinovyev, Ryabitsev, 2000]; Sibirskiye uvaly NP [Zinovyev, Nesterkov, 2003]; Missiya RS, Tobolsk [Bukhkalo et al., 2011]; Kondinskiye lakes NP [Kondinskiye..., 2012].

Material. KHMAO: Surgutsky district (Yugansky NR). AS: Uvatsky (Demyanka), Tobolsky (Verkhniye Aremzyany, Abalak), Vagaysky (Komsomolsky), Nizhnetavdinsky (vicinity of Lake Kuchak, Guzeneevsky reserve, Lake Kultybayka), Tyumensky (Tyumen, vicinity of Lake Andreevskoe, Bogandinsky, Chervishevo, Lake Taraskul) districts.

Distribution. Europe, European Russia, Caucasus, Siberia, Far East, Kazakhstan, Mongolia, Korea, China.

\section{Exocentrini}

*Exocentrus lusitanus (Linnaeus, 1767)

Material. AS: Tyumen, linden plantings, summer 2003, 1 ex., S.D. Sheykin.

Distribution. Europe, European Russia, Caucasus, West Siberia, Kazakhstan.
Tetropini

Tetrops praeustus (Linnaeus, 1758)

Tetrops praeusta: Tobolsk [Bukhkalo et al., 2011].

Material. AS: Tobolsky distr., Verkhniye Aremzyany vill., edge of mixed forest, 4.07.2018, 1 ex., E.V. Sergeeva; Tyumen, 24.07.1992, 1 ex., A.Yu. Dudko, 3.07.1992, 1 ex., A.Yu. Dudko.

Distribution. Europe, European Russia, Caucasus, Siberia, Kazakhstan, Mongolia.

\section{Saperdini}

Saperda (Saperda) carcharias (Linnaeus, 1758)

Saperda carcharias: «Ishimsky district» [Kolosov, 1914]; Tobolsk [Kolosov 1933]; Missiya RS, Tobolsk [Bukhkalo et al., 2011].

Material. AS: Nizhnetavdinsky (vicinity of Lake Kuchak), Tyumensky (Tyumen, Bogandinsky, vicinity of Lake Andreevskoe, Chervishevo, Salairka), Yurginsky (Severo-Pletnyovo), Vikulovsky (Yushkova), Armizonsky (Kalmakskoe), Sladkovsky (Sladkovo) districts.

Distribution. Europe, European Russia, Caucasus, Siberia, Far East, Near East, Kazakhstan, Mongolia, China.

\section{Saperda (Saperda) similis Laicharting, 1784}

Saperda similis: Tobolsk [Kolosov, 1933].

Material. AS: Uvatsky distr., floodplain of the river Alymka at the confluence of the river Rynya, 19.07.2008, 1 ex., V.A. Stolbov, Nizhnetavdinsky distr., vicinity of Lake Kuchak, 19.06.1996, 1 ex., A.N. Mokhratov; 6.2009, 1 ex., unknown collector.

Distribution. Europe, European Russia, Siberia, Far East, Kazakhstan, Central Asia, Mongolia, North China.

\section{Saperda (Lopezcolonia) perforata (Pallas, 1773)}

Saperda perforata: Tobolsk [Bukhkalo et al., 2011].

Material. KHMAO: Khanty-Mansiysk. AS: Uvatsky (Missiya RS), Tobolsky (Abramova, Verkhniye Aremzyany, Ingair), Vagaysky (Kryukovskoe NM), Nizhnetavdinsky (vicinity of Lake Kuchak, Lake Kultybayka), Tyumensky (Bogandinsky, Chervishevo), Zavodoukovsky (Kosheleva) districts.

Distribution. North Africa, Europe, European Russia, Caucasus, Siberia, Far East, Near East, Kazakhstan, Mongolia, China, Japan.

\section{Saperda (Lopezcolonia) scalaris (Linnaeus, 1758)}

Saperda scalaris: Aygunyogan, Lake Taraskul, Dubrovnoe [Sitnikov, 2000]; Tobolsk [Bukhkalo et al., 2011].

Material. AS: Tobolsky (Verkhniye Aremzyany), Yarkovsky (Motushi), Nizhnetavdinsky (vicinity of Lake Kuchak), Tyumensky (Bogandinsky) districts.

Distribution. North Africa, Europe, European Russia, Caucasus, Siberia, Far East, Near East, Kazakhstan, Mongolia, Korea, China.

\section{Saperda (Compsidia) populnea (Linnaeus, 1758)}

Saperda populnea: Krasny kamen station [Olshvang, 1980]; Tobolsk [Bukhkalo et al., 2011].

Compsidia populnea: Rafaylovsky reserve [Bayanov, 2009].

Material. KHMAO: Surgutsky distr., Yugansky NR, 7.08.1994, 3 ex., B.V. Krasutsky, collection of IPAE. AS: Nizhnetavdinsky distr., vicinity of Lake Kultybayka, 6.07.1996, 1 ex., V. Sinitsyn; Tyumensky distr., vicinity of Tyumen, 25.05.1997, 1 ex., P.S. Sitnikov; Yarkovsky distr., vicinity of Lake Bol'shoe Severnoe, mixed forest, 18.06.2019, 1 ex., E.V. Sergeeva.

Distribution. Europe, European Russia, Caucasus, Siberia, Near East, Kazakhstan, Central Asia, Korea, China. 


\section{Menesia sulphurata (Gebler, 1825)}

Menesia sulphurata: Tobolsk [Kolosov, 1936]; Tyumen, Lake Taraskul [Sitnikov, 2000]; Tobolsk, Abramova [Bukhkalo et al., 2011].

Material. AS: Tyumensky distr., vicinity of Voronina vill., 21.06.2007, 1 ex., V.A. Stolbov; Tyumen, Lake Krugloye, 21.06.2003, 1 ex., V.A. Stolbov.

Distribution. South of European Russia, Siberia, Far East, Kazakhstan, Mongolia, China, Korea, Japan.

\section{Phytoeciini}

Oberea (Oberea) oculata (Linnaeus, 1758)

Oberea oculata: Yalutorovsk [Kolosov, 1914]; Tobolsk [Bukhkalo et al., 2011].

Material. AS: Nizhnetavdinsky (vicinity of Lake Kuchak), Tyumensky (Bogandinsky, vicinity of Lake Andreevskoe, Kamenka), Vikulovsky (Tyleshov Bor) districts.

Distribution. North Africa, Europe, European Russia, Caucasus, Siberia, Far East, Near East, Iraq, Kazakhstan, Mongolia, Korea, China.

\section{Phytoecia (Musaria) affinis (Harrer, 1784)}

Phytoecia affinis: Gulyay-Pole [Sitnikov, 2000; Red Data Book..., 2004; Sitnikov et al., 2013].

Material. AS: Kazansky distr., Malye Yarki vill., plot of feather grass steppe on the hillside, 21.06.2009, 1 ex., V.A. Stolbov.

Distribution. Europe, European Russia, Caucasus, West Siberia, Near East, Kazakhstan.

\section{Phytoecia (Phytoecia) nigricornis}

(Fabricius, 1781)

Phytoecia nigricornis: Tyumen [Sitnikov, 2000; Red Data Book..., 2004; Sitnikov et al., 2013].

Distribution. Europe, European Russia, Caucasus, Siberia, Far East, Near East, Kazakhstan.

\section{Phytoecia (Phytoecia) cylindrica (Linnaeus, 1758)}

Phytoecia cylindrica: Tobolsk [Sitnikov, 2000; Lomakin et al., 2001; Red Data Book..., 2004; Alemasova, Galich, 2006; Bukhkalo et al., 2011]; Shorokhovo, Markovo [Sitnikov et al., 2013].

Material. AS: Uvatsky districts (Uvat), Tyumen.

Distribution. Europe, European Russia, Caucasus, Siberia, Far East, Near East, Kazakhstan.

Phytoecia (Phytoecia) virgula (Charpentier, 1825)

Phytoecia virgula: Tobolsk [Bukhkalo et al., 2011].

Material. AS: Tobolsk, Chuvashsky cape, settled slope, 18.06.2012, 1 ex., E.V. Sergeeva.

Distribution. Europe, European Russia, Caucasus, Siberia, Near East, Kazakhstan, Central Asia.

\section{Phytoecia (Phytoecia) icterica (Schaller, 1783)}

Phytoecia icterica: Tyumen [Sitnikov, 2000]; Tyumen, Dubrovnoye [Lomakin et al., 2001; Red Data Book..., 2004].

Material. AS: Tobolsk, 23.05.2015, 1ex., E.V. Sergeeva; Ishimsky distr., Ishimskiye bugry - Gora Lyubvi NM, settled slope, $20.06 .2018-1$ ex., E.V. Sergeeva.

Distribution. Europe, South European Russia, Caucasus, West Siberia, Kazakhstan.

\section{*Phytoecia (Opsilia) coerulescens (Scopoli, 1763)}

Material. AS: Kazansky distr., Novoalexandrovka vill., vicinity of Lake Siverga, forb meadow, on Lithospermum officinale L., 4-6.06.2019, 5 ex., E.V. Sergeeva.
Distribution. North Africa, Europe, European Russia, Caucasus, West Siberia, Kazakhstan, Central Asia.

Agapanthiini

Agapanthia (Epoptes) dahli C. F.W. Richter, 1821

Agapanthia dahli: Uspenka, Shashova, Ostropyatovo, Travnoe [Sitnikov, 2000; Sitnikov et al., 2004]; Abalak [Bukhkalo et al., 2011].

Material. AS: Vagaysky (Kryukovskoe NM), Nizhnetavdinsky (vicinity of Lake Kuchak), Zavodoukovsky (Gilyovo), Isetsky (Bityuki), Ishimsky (Sazhino), Berdyuzhsky (Okunyovo, Polovinnoe), Kazansky (Afonkino), Sladkovsky (Mayka, Island Tavolzhan) districts.

Distribution. Europe, European Russia, Caucasus, Siberia, Kazakhstan, Central Asia.

\section{Agapanthia (Epoptes) villosoviridescens} (DeGeer, 1775)

Agapanthia lineatocollis: Yalutorovsk [Kolosov, 1914]. Agapanthia villosoviridescens: Tobolsk, Abalak, Vinokurova [Bukhkalo et al., 2011].

Material. KHMAO: Surgutsky district (Yugansky NR, collection of IPAE). AS: Tobolsky (Ovsyannikova, Penya), Yarkovsky (Dubrovnoe), Nizhnetavdinsky (vicinity of Lake Kuchak), Tyumensky (Tyumen, Bogandinsky, Lake Lukashinskoye, Yantyk), Yalutorovsky (Lake Singul, Moshkarinsky reserve, Novoatyalovo), Isetsky (Bityuki), Omutinsky (Dmitrievka) districts.

Distribution. Europe, European Russia, Caucasus, Siberia, Kazakhstan, Mongolia.

\section{Agapanthiola leucaspis (Steven, 1817)}

Agapanthia leucaspis: Tyumen; Tavolzhan [Sitnikov, 2000; Sitnikov et al., 2004].

Material. AS: Ishimsky distr., Ishimskiye bugry - Gora Lyubvi NM, settled slope, 20.06.2018, 7 ex., E.V. Sergeeva; Berdyuzhsky distr., Okunyovo vill., steppe meadow, 10.07.2018, 1ex., E.V. Sergeeva; Armizonsky distr., Poloe vill., steppe meadow, 30.07.2019, 1 ex., E.V. Sergeeva; Kazansky distr., Afonkino vill., 6.2009, 2 ex., S.A. Ivanov; Novoalexandrovka vill., 12.07.2018, 1ex., E.V. Sergeeva; vicinity of Lake Siverga, 4-6.06.2019, 3 ex., E.V. Sergeeva; Sladkovsky distr., Island Tavolzhan, steppe meadow, 21.06.2018, 2 ex., E.V. Sergeeva.

Distribution. Europe, European Russia, Caucasus, Siberia, Near East, Kazakhstan, Central Asia, Mongolia.

\section{Conclusion}

Thus, on the territory of the Tyumen oblast 99 species from 59 genera of Longicorn beetles are registered. Of these, 28 species are known from the Yamalo-Nenets Autonomous Okrug, located in the tundra, forest-tundra and northern taiga zones, 52 - from the KhantyMansi Autonomous Okrug (mostly middle taiga) and 85 - from the administrative south of the region (southern taiga, subtaiga and forest-steppe zones).

Eleven species, Dinoptera collaris (Linnaeus, 1758), Pachytodes erraticus (Dalman, 1817), Stenurella bifasciata (Müller, 1776), Tetropium gracilicorne Reitter, 1889, Spondylis buprestoides (Linnaeus, 1758), Pronocera sibirica (Gebler, 1848), Semanotus undatus (Linnaeus, 1758), Monochamus impluviatus (Motschulsky, 1859), Lamia textor (Linnaeus, 1758), Exocentrus lusitanus (Linnaeus, 1767) and and Phytoecia coerulescens (Scopoli, 1763) are recorded for the region for the first time. 
From the list of species previously cited for the Tyumenskaya oblast [Chugunov, 1917; Kolosov, 1933; Plavilstshikov, 1936; Sitnikov, 1998, 2000; Bukhkalo et al., 2011; Tolstikov et al., 2013] Cornumutila quadrivittata Gebler, 1830, Anastrangalia dubia (Scopoli, 1763), Obrium brunneum (Fabricius, 1793), Purpuricenus kaehleri (Linnaeus, 1758) and Eodorcadion carinatum (Fabricius, 1781) are excluded, due to erroneous determination and questionable label data.

Nine species, Rhagium sycophanta (Schrank, 1781), Cornumutila lineata (Letzner, 1844), Nivellia extensa (Gebler, 1833), Anastrangalia renardi (Gebler, 1848), Judolia dentatofasciata (Mannerheim, 1852), Tetropium aquilonium Plavilstshikov, 1940, Hylotrupes bajulus (Linnaeus, 1758), Callidium aeneum (DeGeer, 1775) and Acanthocinus carinulatus (Gebler, 1833) are known only from literature.

Seven species: Tragosoma depsarium (Linnaeus, 1767), Stenocorus meridianus (Linnaeus, 1758), Purpuricenus globulicollis Mulsant, 1839, Aromia moschata (Linnaeus, 1758), Chlorophorus herbstii (Brahm, 1790), Echinocerus floralis (Pallas, 1773) and Xylotrechus pantherinus (Savenius, 1825) are included in the list of species of animals, plants and mushrooms to be entered in the Red Data Book of the Tyumenskaya oblast [2017].

\section{Acknowledgements}

The authors are sincerely grateful to M.L. Danilevsky (A.N. Severtsov Institute of Ecology and Evolution of the Russian Academy of Sciences, Moscow) for help in identification of doubtful species and valuable advice in the process of preparation of the manuscript. The authors are thankful to P.S. Sitnikov, S.A. Ivanov, D.E. Galich (Tyumen) and to all collectors, who kindly provided their materials for research, as well as to N.G. Erokhin (Museum of Institute of Plant and Animal Ecology of the Russian Academy of Sciences, Yekaterinburg) and O.I. Marakulina (Museum Complex of Slovtsov, Tyumen) for assistance in a loan of the museum collections.

\section{References}

Alemasova N.V., Galich D.E. 2006. [Addition to the list of species of Tobolsk district listed in the Red Data Book of the Tyumen region] // Vestnik Tobolskogo gosudarstvennogo pedagogicheskogo instituta. No.6. P.179-187. [In Russian].

Bayanov E.S. 2009. [The fauna of the reserve «Rafaylovsky reserve» (south of the Tyumen region)] // VII Zyryanovskiye chteniya: Materialy Vserossiyskoy nauchno-prakticheskoy konferentsii Kurgan. P.240-242. [In Russian].

Bukhkalo S.P., Galich D.E., Sergeeva E.V., Alemasova N.V. 2011. [Synopsis beetle fauna of the southern taiga of Western Siberia (in the basin of the lower Irtysh)]. Moscow: KMK. 267 p. [In Russian].

Cherepanov A.I. 1970. [The fauna of Longicorn beetles (Coleoptera, Cerambycidae) of Siberia] // Fauna of Siberia. Novosibirsk: Nauka. P.101-122. [In Russian].

Cherepanov A.I. 1979. [Longicorn beetles of North Asia. Part 1 (Prioninae, Disteniinae, Lepturinae, Aseminae).] Novosibirsk: Nauka. 470 p. [In Russian].

Cherepanov A.I. 1981. [Longicorn beetles of North Asia. Part 2 (Cerambycinae).] Novosibirsk: Nauka. 216 p. [In Russian].
Cherepanov A.I. 1982. [Longicorn beetles of North Asia. Part 3 (Cerambycinae: Clytini, Steaspini).] Novosibirsk: Nauka. 257 p. [In Russian].

Cherepanov A.I. 1983. [Longicorn beetles of North Asia. Part 4 (Lamiinae: Dorcadionini - Apomecynini).] Novosibirsk: Nauka. 223 p. [In Russian].

Cherepanov A.I. 1984. [Longicorn beetles of North Asia. Part 5 (Lamiinae: Pterycoptini — Agapanthiini).] Novosibirsk: Nauka. 214 p. [In Russian].

Cherepanov A.I. 1985. [Longicorn beetles of North Asia. Part 6 (Lamiinae: Saperdini — Tetraopini)] Novosibirsk: Nauka. 256 p. [In Russian].

Chugunov S.M. 1917. [From Tobolsk to Obdorsk in the summer of 1915] // Yezhegodnik Tobolskogo gubernskogo muzeya. No.28. P.1-18. [In Russian].

Csíki E. 1901. Coleopteren // Dritte Asiatische Forschungsreise des Grafen Eugen Zichy. Band II. Budapest - Leipzig. P.75-120.

Danilevsky M.L. 2014. [Longicorn beetles (Coleoptera, Cerambycoidea) of Russia and adjacent countries] Part 1. Moscow: HSC. 522 p. [In Russian].

Danilevsky M.L. 2019. A check list of the longicorn beetles (Cerambycoidea) of Russia // http://www.cerambycidae.net/ russia.pdf.

Danilevsky M.L., Dedyukhin S.V., Egorov L.V., Kadyrbekov R.Kh., Kasatkin D.G., Shapovalov A.M. 2007. Purpuricenus globulicollis Dejean in Mulsant, 1839 - A Mediterranian longicorn-beetle (Coleoptera: Cerambycidae) in the fauna of Russia and Kazakhstan // Russian Entomological Journal. Vol.16. No.1. P.63-69.

Fridolin V.Yu. 1935. [The fauna of the Northern Urals as a zoogeographical unit and as a biocenotic whole] // Trudy lednikovykh ekspeditsiy. Leningad. Vol.4. P.245-270. [In Russian].

Galich D.E. 2016. [About new finds of the Red Book insect species (Insecta) in the Tyumen region] // Sovremennyye tendentsii razvitiya nauki i tekhnologiy. No.10. Pt.3. P.7173. [In Russian].

Galich D.E., Sitnikov P.S. 2015. [Changes and additions to the List of protected species of invertebrate animals of the Tyumen region and its Application] // Nauchnyye vedomosti Belgorodskogo gosudarstvennogo universiteta. Seriya yestestvennyye nauki. Vol.15(212). No.32. P.94-100. [In Russian].

Gerasimov A.G., Gerasimova A.A., Subbotin A.M. 2015. [Overview of the entomofauna of the vicinity of the village of Batovo, Khanty-Mansi Autonomous Okrug] // Arktika, subarktika: mozaichnost, kontrastnost, variativnost kriosfery. Trudy mezhdunarodnoy konferentsii. Tyumen. P.62-64. [In Russian].

Jacobson G.G. 1910. [To the systematics and geographical distribution of species of the genus Monochamus of the Russian fauna] // Proceedings of the Russian Entomological Society. Vol.39. P.489-507. [In Russian].

Kiseleva E.F. 1926. [About beetles (Coleoptera Cerambycidae) near Tomsk] // Izvestiya Tomskogo gosudarstvennogo universiteta. Vol.77. No.2. P.123-133. [In Russian].

Kolosov Yu.M. 1914. [A note on insects of the Tobolsk province] // Zapiski Uralskogo obshchestva lyubiteley yestestvoznaniya. Yekaterinburg. Vol.34. No.1-2. P.1336. [In Russian].

Kolosov Yu.M. 1928. [Several brief notes about insects of Western Siberia] // Izvestiya gosudarstvennogo ZapadnoSibirskogo muzeya. Omsk. Vol.1. P.75-76. [In Russian].

Kolosov Yu.M. 1933. [Materials for the insect fauna of the Tobolsk North] // Trudy Uralskogo oblastnogo instituta mikrobiologii i epidemiologii. Vol.1. No.1. SverdlovskMoscow. P.49-58. [In Russian].

Kolosov Yu.M. 1936. [Insects of the Urals] // Nature of the Urals. Sverdlovsk. P.233-251. [In Russian]. 
Koltunov E.V., Zinovyev E.V., Zalesov S.V., Gilev A.V. 2009 [Flora and fauna of the Samarovsky Chugas Natural Park. Entomofauna]. Yekaterinburg. 178 p. [In Russian].

[Kondinskiye Lakes Natural Park. 2012] // V.M. Kalinin (eds.). Yekaterinburg. 398 p. [In Russian].

Korshunov Yu.P. 1973. [To the entomofauna of the north of the middle taiga of Western Siberia] // Priroda taygi Zapadnoy Sibiri. Novosibirsk: Nauka. P.136-151. [In Russian].

Krasutsky B.V., Pekin V.P. 2014. [Insects of the Red Data Book of the Tyumen region in the projected protected areas] // Ekologicheskiy monitoring i bioraznoobraziye. No.2(9). P.76-81. [In Russian].

Kuleshov D.A., Romanenko V.N. 2009. [Longicorn beetles (Coleoptera, Cerambycidae) of the Tomsk region] // Vestnik Tomskogo gosudarstvennogo universiteta. Biologiya. No.4(8). P.29-40. [In Russian].

Lazarev M.A. 2009. Cornumutila quadrivittata (Gebler, 1830) and C. lineata (Letzner, 1844), stat. rest. (Coleoptera, Cerambycidae) from Western Europe and Russia // Spec. Bull. Jpn. Soc. Coleopterol. Tokyo. Vol.7. P.117-126.

Lomakin D.E., Menshchikov A.G., Sitnikov P.S., Sharapova T.A. 2001. [On the criteria for the selection of insects for inclusion in the Red Data Book of the Tyumen region. New faunal finds] // Yezhegodnik Tyumenskogo oblastnogo krayevedcheskogo muzeya: 2000. Tyumen. P.349-366. [In Russian].

Obogrelov A.A., Sitnikov P.S., Khozyainova N.V. 2002. Features of flora and entomofauna of Isetsky region (according to the results of expeditions of 2000) // Yezhegodnik Tyumenskogo oblastnogo krayevedcheskogo muzeya: 2001. Tyumen. P.338-352. [In Russian].

Olshvang V.N. 1977. [Biomass and population dynamics of arthropod of mesofauna in Priobye forest-tundra] // Biotsenoticheskaya rol' zhivotnykh $\mathrm{v}$ lesotundre Yamala. Sverdlovsk. P.31-71. [In Russian].

Olshvang V.N. 1980. [Insects of the Polar Urals and Priobye forest-tundra] // Fauna i ekologiya nasekomykh Priobskogo Severa. Sverdlovsk. P.3-37. [In Russian].

Olshvang V.N. 1992. [The structure and dynamics of the insect population of South Yamal]. Yekaterinburg: Nauka. 104 p. [In Russian].

Plavilstshikov N.N. 1915a. Les especes palearctiques du genre Rhagium F. (Coleoptera, Cerambycidae) // Revue Russ. d'Entom. Vol.15. No.1. P.31-49. [In Russian].

Plavilstshikov N.N. 1915b. Especes eurasiques du genre Evodinus J. Lec. (Coleoptera, Cerambycidae) // Revue Russ. d‘Entom. Vol.15. No.3. P.354-382. [In Russian].

Plavilstshikov N.N. 1930. [Palaearctic species of the tribus Asemini (Coleoptera, Cerambycidae)] // Izvestiya Uralskogo Politekhnicheskogo Instituta. No.7. P.181-187. [In Russian].

Plavilstshikov N.N. 1936. Faune de l'URSS. Insects Coléptères. Vol.21. Cerambycidae (Pt.1). M.-L. 612 p. [In Russian].

Plavilstshikov N.N. 1940. Faune de l'URSS. Insects Coléptères. Vol.22. Cerambycidae (Pt.2). M.-L. 785 p. [In Russian].

[Red Data Book of the Tyumen region: Animals, plants and mushrooms. 2004]. Yekaterinburg: Publisher of the Urals university. 496 p. [In Russian].

Redikortsev V.V. 1908. [Materials to the entomofauna of the Urals] // Zapiski Uralskogo obshchestva lubiteley estestvoznaniya. No.27. P.95-122. [In Russian].

RyabitsevA.A. 1997. [Fauna of the North Yamal's beetles] // Uspekhi entomologii na Urale. Yekaterinburg. P. 85-88. [In Russian].

Samko K.P. 1928a. [To the knowledge of the entomofauna of the Tobolsk District] // Byulleten Obshchestva izucheniya kraya pri Muzeye Tobolskogo severa. No.1(2). P.17-24. [In Russian].
Samko K.P. 1928b. [About a trip with an entomological goal in the summer of 1928 to the river Shchuchya of the Tobolsk North] // Byulleten Obshchestva izucheniya kraya pri Muzeye Tobolskogo severa. No.3(4). P.7-12. [In Russian].

Shapovalov A.M. 2012. [Longhorn beetle (Coleoptera, Cerambycidae) of Orenburg region: fauna, distribution, bionomics] // Trudy Orenburgskogo otdeleniya Russkogo entomologicheskogo obshchestva. Orenburg. No.3. 221 p. [In Russian].

Shukhov I.N. 1914. [The Shchuchya River. Geographical description of the river and travel to its valley in 1913] // Yezhegodnik Tobol'skogo gubernskogo muzeya. Vol.22. P.1-31. [In Russian].

Sitnikov P.S. 1998. [Rare insects of the south of the Tyumen region] // Yezhegodnik Tyumenskogo oblastnogo krayevedcheskogo muzeya: 1995. Tyumen. P.128-139. [In Russian].

Sitnikov P.S. 2000. [Rare finds of the Longicorn beetles from the Tyumen collections] // Yezhegodnik Tyumenskogo oblastnogo krayevedcheskogo muzeya: 1999. Tyumen. P.216-224. [In Russian].

Sitnikov P.S. 2002. [Expedition "The Red Book-2002": Entomological discoveries] // Slovtsovskiye chteniya. Tyumen. P.239-241. [In Russian].

Sitnikov P.S. 2010. [New species of rare insects of the Tyumen region] // Zemlya Tyumenskaya. Vol.22. Tyumen. P.328333. [In Russian].

Sitnikov P.S. 2013. [New records of rare insects from the Red Data Book of the Tyumen region] // Materialy ko vtoromu izdaniyu Krasnoy knigi Tyumenskoy oblasti. Tyumen: OOO «TyumenNIIgiprogaz». P.95-100. [In Russian].

Sitnikov P.S. Lomakin D.E., Sharapova T.A. 2004. [Rare species of invertebrate animals of the 4th category in the Red Data Book of the Tyumen Region] // Zemlya Tyumenskaya: Yezhegodnik Tyumenskogo oblastnogo krayevedcheskogo muzeya: 2003. Vol.17. Tyumen: TSU. P.269-292. [In Russian].

Sitnikov P.S., Lomakin D.E., Ivanov S.A. 2013. [Proposals on the correction of the main list of rare species of invertebrates in the Red Data Book of the Tyumen Region] // Ekologiya zhivotnykh i faunistika: Sbornik nauchnykh trudov Kafedry Zoologii i Evolyutsionnoy Ekologii Zhivotnykh. No.9. Tyumen: TSU. P.11-137. [In Russian].

Stolbov V.A., Galich D.E., Lomakin D.E. 2017. [New data on rare insects and arachnids of the Tyumen region] // Vestnik Tyumenskogo gosudarstvennogo universiteta. Ekologiya i prirodopolzovaniye. Vol.3. No.2(10). P.81-94. [In Russian].

Striganova B.R., Poryadina N.M. 2005. [Animal population of the soils of the boreal forests of the West Siberian Plain]. M.: KMK. 234 p. [In Russian].

Tatarinova A.F., Nikitsky N.B., Dolgin M.M. 2007. [Longicorn beetles (Coleoptera, Cerambycidae). Fauna of the European Northeast of Russia]. SPb.: Nauka. Vol.8. Part 2. 301 p. [In Russian].

[The list of species of animals, plants and mushrooms to be entered in the Red Data Book of the Tyumen region: Annex to the decree of the Government of the Tyumen region from 14.04.2017 No.145-p. [In Russian].

Tolstikov A.V., Kuzmin I.V., Stolbov V.A. 2013. [Protected invertebrates of the Tyumen region in the collection of the Zoological Museum of the Tyumen University] // Ekologiya zhivotnykh i faunistika: Sbornik nauchnykh trudov Kafedry Zoologii i Evolyutsionnoy Ekologii Zhivotnykh. No.9. Tyumen: TSU. P.161-167. [In Russian].

Ukhova N.L. 2010. [Specially protected species of invertebrates in the territory of the Kondinskiye lakes natural park] // 
Okhrana, ekoprosveshcheniye, rekreatsionnaya deyatel'nost', izucheniye bioraznoobraziya i kul'turnogo naslediya na OOPT. Materialy regional'noy nauchnoprakticheskoy konferentsii. Nizhny Tagil. P.132-136. [In Russian].

Zinovyev E.V. 2005. [Data on the beetle's fauna of the Samarovsky Chugas Natural Park] // Biologicheskiye resursy i prirodopolzovaniye. Surgut. Vol.8. P.90-113. [In Russian].

Zinovyev E.V. 2008. [New data on the beetle fauna (Insecta: Coleoptera) of the Samarovsky Chugas Natural Park] // Biologicheskiye resursy i prirodopolzovaniye. Surgut. Vol.11. P.182-201. [In Russian].

Zinovyev E.V., Ryabitsev A.V. 2000. [To the fauna of beetles of Siberian Uvaly (Western Siberia)] // Materialy k poznaniyu fauny i flory Yamalo-Nenetskogo avtonomnogo okruga.
Nauchnyy vestnik. Salekhard. Vol.4(II). P.51-55. [In Russian].

Zinovyev E.V., Malozemov A.Yu. 2002. [Fauna of herpetobiont beetles of the Neroika Mountain (Polar Urals)] // Sibirskiy ekologicheskiy zhurnal. Vol. 9. No.6. P.703-710. [In Russian].

Zinovyev E.V., Nesterkov A.V. 2003. [Species composition of beetles (Insecta: Coleoptera) of the Siberian Uvaly Conservation and Natural Park] // Ekologicheskiye issledovaniya vostochnoy chasti Sibirskikh Uvalov: Sbornik nauchnykh trudov ZPP Sibirskiye Uvaly. Nizhnevartovsk: Priobye publishers. P.83-118. [In Russian].

Zinovyev E.V., Olshvang V.N. 2003. [The beetles of the north of the West Siberian Plain, the Subpolar and Polar Urals] // Biologicheskiye resursy Polyarnogo Urala. Nauchnyy vestnik. Salekhard. Vol.3(II). P.37-60. [In Russian].

Поступила в редакцию 7.5.2019 